\title{
On the Role of Information Theoretic Uncertainty Relations in Quantum Theory
}

\author{
Petr Jizba* \\ FNSPE, Czech Technical University in Prague, \\ Břehová 7, 11519 Praha 1, Czech Republic \\ and \\ ITP, Freie Universität Berlin, \\ Arnimallee 14 D-14195 Berlin, Germany \\ Jacob A. Dunningham \\ Department of Physics and Astronomy, \\ University of Sussex, Falmer, \\ Brighton, BN1 9QH UK \\ Jaewoo Jod \\ School of Physics and Astronomy, \\ University of Leeds, Leeds LS2 9JT, UK
}

\begin{abstract}
Uncertainty relations based on information theory for both discrete and continuous distribution functions are briefly reviewed. We extend these results to account for (differential) Rényi entropy and its related entropy power. This allows us to find a new class of information-theoretic uncertainty relations (ITURs). The potency of such uncertainty relations in quantum mechanics is illustrated with a simple two-energy-level model where they outperform both the usual RobertsonSchrödinger uncertainty relation and Kraus-Maassen Shannon entropy based uncertainty relation. In the continuous case the ensuing entropy power uncertainty relations are discussed in the context of heavy tailed wave functions and Schrödinger cat states. Again, improvement over both the Robertson-Schrödinger uncertainty principle and Shannon ITUR is demonstrated in these cases. Further salient issues such as the proof of a generalized entropy power inequality and a geometric picture of information-theoretic uncertainty relations are also discussed.
\end{abstract}

PACS numbers: 03.65.-w; 89.70.Cf

Keywords: Information-theoretic Uncertainty Relations; Rényi Entropy; Entropy-power Inequality; Quantum Mechanics

*Electronic address: p.jizba@fjfi.cvut.cz

${ }^{\dagger}$ Electronic address: J.Dunningham@sussex.ac.uk

${ }^{\ddagger}$ Electronic address: J.Joo@leeds.ac.uk 


\section{INTRODUCTION}

Quantum-mechanical uncertainty relations place fundamental limits on the accuracy with which one is able to measure the values of different physical quantities. This has profound implications not only on the microscopic but also on the macroscopic level of physical systems. The archetypal uncertainty relation formulated by Heisenberg in 1927 describes a trade-off between the error of a measurement to know the value of one observable and the disturbance caused on another complementary observable so that their product should be no less than a limit set by $\hbar$. Since Heisenberg's intuitive, physically motivated deduction of the error-disturbance uncertainty relations [1, 2], a number of methodologies trying to improve or supersede this result have been proposed. In fact, over the years it have became steadily clear that the intuitiveness of Heisenberg's version cannot substitute mathematical rigor and it came as no surprise that the violation of the Heisenberg's original relation was recently reported a number of experimental groups, e.g., most recently by the Vienna group in neutron spin measurements [3]. At present it is Ozawa's universally valid error-disturbance relation [35, 36] that represents a viable alternative to Heisenberg's error-disturbance relation.

Yet, already at the end of 1920s Kennard and independently Robertson and Schrödinger reformulated the original Heisenberg (single experiment, simultaneous measurement, errordisturbance) uncertainty principle in terms of a statistical ensemble of identically prepared

experiments [4 6]. Among other things, this provided a rigorous meaning to Heisenberg's imprecisions ("Ungenauigkeiten") $\delta x$ and $\delta p$ as standard deviations in position and momenta, respectively, and entirely avoided the troublesome concept of simultaneous measurement. The Robertson-Schrödinger approach has proven to be sufficiently versatile to accommodate other complementary observables apart from $x$ and $p$, such as components of angular momenta, or energy and time. Because in the above cases the variance is taken as a "measure of uncertainty", expressions of this type are also known as variance-based uncertainty relations (VUR). Since Robertson and Schrödinger's papers, a multitude of VURs has been devised; examples include the Fourier-type uncertainty relations of Bohr and Wigner [17, 18], the fractional Fourier-type uncertainty relations of Mustard [19], mixed-states uncertainty relations [29], the angle-angular momentum uncertainty relation of Lévy-Leblond [30] and Carruthers and Nietto [31], the time-energy uncertainty relation of Mandelstam and Tamm [32], Luisell's amplitude-phase uncertainty relation [33], and Synge's three-observable uncertainty relations [34].

Many authors [7 9, 12 14] have, however, remarked that even VURs have many limitations. In fact, the essence of a VUR is to put an upper bound to the degree of concentration of two (or more) probability distributions, or, equivalently impose a lower bound to the associated uncertainties. While the variance is often a good measure of the concentration of a given distribution, there are many situations where this is not the case. For instance, variance as a measure of concentration is a dubious concept in the case when a distribution contains more than one peak. Besides, variance diverges in many distributions even though such distributions are sharply peaked. Notorious examples of the latter are provided by heavy-tail distributions such as Lévy [10, 11], Weibull [11] or Cauchy-Lorentz distribu- 
tions [11, 15]. For instance, in the theory of Bright-Wigner shapes it has been known for a long time [16] that the Cauchy-Lorentz distribution can be freely concentrated into an arbitrarily small region by changing its scale parameter, while its standard deviation remains very large or even infinite.

Another troublesome feature of VURs appears in the case of finite-dimensional Hilbert spaces, such as the Hilbert space of spin or angular momentum. The uncertainty product can attain zero minimum even when one of the distributions is not absolutely localized, i.e., even when the value of one of the observables is not precisely known [8]. In such a case the uncertainty is just characterized by the lower bound of the uncertainty product (i.e., by zero) and thus it only says that this product is greater than zero for some states and equal to zero for others. This is, however, true also in classical physics.

The previous examples suggest that it might be desirable to quantify the inherent quantum unpredictability in a different, more expedient way. A distinct class of such nonvariance-based uncertainty relations are the uncertainty relations based on information theory. In these the uncertainty is quantified in terms of various information measures entropies, which often provide more stringent bound on concentrations of the probability distributions. The purpose of the present paper is to give a brief account of the existing information-theoretic uncertainty relations (ITUR) and present some new results based on Rényi entropy. We also wish to promote the notion of Rényi entropy (RE) which is not yet sufficiently well known in the physics community.

Our paper is organized in the following way: In Section III, we provide some informationtheoretic background on the Rényi entropy (RE). In particular, we stress distinctions between the RE for discrete probabilities and RE for continuous probability density functions (PDF) - the so-called differential RE. In Section III we briefly review the concept of entropy power both for Shannon and Rényi entropy. We also prove the generalized entropy power inequality. With the help of the Riesz-Thorin inequality we derive in Section IV the RE-based ITUR for discrete distributions. In addition, we also propose a geometric illustration of the latter in terms of the condition number and distance to singularity. In Section $\mathrm{V}$ we employ the Beckner-Babenko inequality to derive a continuous variant of the RE-based ITUR. The result is phrased both in the language of REs and generalized entropy powers. In particular, the latter allows us to establish a logical link with the Robertson-Schrödinger VUR. The advantage of ITURs over the usual VUR approach is illustrated in Section VI, In two associated subsections we first examine the rôle of a discrete generalized ITUR on a simple two-level quantum system. In the second subsection the continuous ITUR is considered for quantum-mechanical systems with heavy-tailed distributions and Schrödinger cat states. An improvement of the Rényi ITUR over both the Robertson-Schrödinger VUR and Shannon ITUR is demonstrated in all the cases discussed. Finally in Section VII we make some concluding remarks and propose some generalizations. For the reader's convenience we relegate to Appendix A some of the detailed mathematical steps needed in Sections IIIA and $\mathrm{V}$ 


\section{BRIEF INTRODUCTION TO RÉNYI ENTROPY}

The basic notion that will be repeatedly used in the following sections is the notion of Rényi entropy. For this reason we begin here with a brief review of some of its fundamental properties.

REs constitute a one-parameter family of information entropies labeled by Rényi's parameter $\alpha \in \mathbb{R}^{+}$and fulfill additivity with respect to the composition of statistically independent systems. The special case with $\alpha=1$ corresponds to the familiar Shannon entropy. It can be shown that Rényi entropies belong to the class of mixing homomorphic functions [20] and that they are analytic for $\alpha$ 's which lie in $I \cup I V$ quadrants of the complex plane [21]. In order to address the uncertainty relations issue it is important to distinguish two situations.

\section{A. Discrete probability distribution case}

Let $\mathcal{X}=\left\{x_{1}, \ldots, x_{n}\right\}$ be a random variable admitting $n$ different events (be it outcomes of some experiment or microstates of a given macrosystem), and let $\mathcal{P}=\left\{p_{1}, \ldots, p_{n}\right\}$ be the corresponding probability distribution. Information theory then ensures that the most general information measures (i.e. entropy) compatible with the additivity of independent events are those of Rényi [22]:

$$
\mathcal{I}_{\alpha}(\mathcal{P})=\frac{1}{(1-\alpha)} \log _{2}\left(\sum_{k=1}^{n} p_{k}^{\alpha}\right) .
$$

Form (1) is valid even in the limiting case when $n \rightarrow \infty$. If, however, $n$ is finite then Rényi entropies are bounded both from below and from above: $\log _{2}\left(p_{k}\right)_{\max } \leq \mathcal{I}_{\alpha} \leq \log _{2} n$. In addition, REs are monotonically decreasing functions in $\alpha$, so $\mathcal{I}_{\alpha_{1}}<\mathcal{I}_{\alpha_{2}}$ if and only if $\alpha_{1}>\alpha_{2}$. One can reconstruct the entire underlying probability distribution knowing all Rényi distributions via the Widder-Stiltjes inverse formula [21]. In this case the leading order contribution comes from $\mathcal{I}_{1}(\mathcal{P})$, i.e. from Shannon's entropy. Some elementary properties of $\mathcal{I}_{\alpha}$ are as follows:

1. RE is symmetric: $\mathcal{I}_{\alpha}\left(p_{1}, \ldots, p_{n}\right)=\mathcal{I}_{\alpha}\left(p_{k(1)}, \ldots, p_{k(n)}\right)$.

2. RE is nonnegative: $\mathcal{I}_{\alpha}(\mathcal{P}) \geq 0$.

3. $\mathrm{RE}$ is decisive: $\mathcal{I}_{\alpha}(0,1)=\mathcal{I}_{\alpha}(1,0)$.

4. For $\alpha \leq 1 \mathrm{RE}$ is concave; for $\alpha>1 \mathrm{RE}$ in neither convex nor concave.

5. RE is bounded, continuous and monotonic in $\alpha$.

6. $\mathrm{RE}$ is analytic in $\alpha \in \mathbb{C}_{I \cup I I I} \Rightarrow$ for $\alpha=1$ it equals to Shannon's entropy, i.e. $\lim _{\alpha \rightarrow 1} \mathcal{I}_{\alpha}=\mathcal{H}$. 
Among a myriad of information measures REs distinguish themselves by having a firm operational characterization in terms of block coding and hypotheses testing. Rényi's parameter $\alpha$ is then directly related to so-called $\beta$-cutoff rates [25]. RE is used in coding theory [26, 27], cryptography [28, 37, 38], finance [39, 40] and in theory of statistical inference 22. In physics one often uses $\mathcal{I}_{\alpha}(\mathcal{P})$ in the framework of quantum information theory [38, 41, 42].

\section{B. Continuous probability distribution case}

Let $M$ be a measurable set on which is defined a continuous probability density function (PDF) $\mathcal{F}(\mathbf{x})$. We will assume that the support (or outcome space) is a smooth but not necessarily compact manifold. By covering the support with the mesh $M^{(l)}$ of $d$-dimensional (disjoint) cubes $M_{k}^{(l)}(k=1, \ldots, n)$ of size $l^{d}$ we may define the integrated probability in $k$-th cube as

$$
p_{n k}=\mathcal{F}\left(\mathbf{x}_{i}\right) l^{d}, \quad \mathbf{x}_{i} \in M_{k}^{(l)} .
$$

This defines the mesh probability distribution $\mathcal{P}_{n}=\left\{p_{n 1}, \ldots, p_{n n}\right\}$. Infinite precision of measurements (i.e., when $l \rightarrow 0$ ) often brings infinite information. As the most "junk" information comes from the uniform distribution $\mathcal{E}_{n}$, it is more sensible to consider the relative information entropy rather than absolute one. In references [21, 22] it was shown that in the limit $n \rightarrow \infty$ (i.e., $l \rightarrow 0$ ) it is possible to define a finite information measure compatible with information theory axioms. This renormalized Rényi entropy, often known as differential RE entropy, reads

$$
\tilde{\mathcal{I}}_{\alpha}(\mathcal{F}) \equiv \lim _{n \rightarrow \infty}\left(\mathcal{I}_{\alpha}\left(\mathcal{P}_{n}\right)-\mathcal{I}_{\alpha}\left(\mathcal{E}_{n}\right)\right)=\frac{1}{(1-\alpha)} \log _{2}\left(\frac{\int_{M} d \mathbf{x} \mathcal{F}^{\alpha}(\mathbf{x})}{\int_{M} d \mathbf{x} 1 / V^{\alpha}}\right) .
$$

Here $V$ is the volume of $M$. Equation (3) can be viewed as a generalization of the KullbackLeibler relative entropy [43]. When $M$ is compact it is possible to introduce a simpler alternative prescription as

$$
\begin{aligned}
\mathcal{I}_{\alpha}(\mathcal{F}) & \equiv \lim _{n \rightarrow \infty}\left(\mathcal{I}_{\alpha}\left(\mathcal{P}_{n}\right)-\left.\mathcal{I}_{\alpha}\left(\mathcal{E}_{n}\right)\right|_{V=1}\right)=\lim _{n \rightarrow \infty}\left(\mathcal{I}_{\alpha}\left(\mathcal{P}_{n}\right)+D \log _{2} l\right) \\
& =\frac{1}{(1-\alpha)} \log _{2}\left(\int_{M} d \mathbf{x} \mathcal{F}^{\alpha}(\mathbf{x})\right) .
\end{aligned}
$$

In both previous cases $D$ represents the Euclidean dimension of the support. Rényi entropies (3) and (44) are defined if (and only if) the corresponding integral $\int_{M} d \mathbf{x} \mathcal{F}^{\alpha}(\mathbf{x})$ exists. Equations (3) and (4) indicate that the asymptotic expansion for $\mathcal{I}_{\alpha}\left(\mathcal{P}_{n}\right)$ has the form:

$$
\mathcal{I}_{\alpha}\left(\mathcal{P}_{n}\right)=-D \log _{2} l+\mathcal{I}_{\alpha}(\mathcal{F})+o(1)=-D \log _{2} l+\tilde{\mathcal{I}}_{\alpha}(\mathcal{F})+\log _{2} V_{n}+\mathcal{O}(1) .
$$

Here $V_{n}$ is the covering volume and the symbol $\mathcal{O}(1)$ is the residual error which tends to 0 for $l \rightarrow 0$. In contrast to the discrete case, Rényi entropies $\mathcal{I}_{\alpha}(\mathcal{F})$ are not generally positive. In particular, a distribution which is more confined than a unit volume has less $\mathrm{RE}$ than the corresponding entropy of a uniform distribution over a unit volume and hence yields a negative $\mathcal{I}_{\alpha}(\mathcal{F})$. A paradigmatic example of this type of behavior is the $\delta$-function 
PDF in which case $\mathcal{I}_{\alpha}=-\log _{2} \delta(0)=-\infty$, for all $\alpha$. Information measures $\tilde{\mathcal{I}}_{\alpha}(\mathcal{F})$ and $\mathcal{I}_{\alpha}(\mathcal{F})$ are often applied in theory of statistical inference [44 47] and in chaotic dynamical systems [48, 51$]$.

\section{ENTROPY POWER AND ENTROPY POWER INEQUALITIES}

The mathematical underpinning for most uncertainty relations used in quantum mechanics lies in inequality theory. For example, the wave-packet uncertainty relations are derived from the Plancherel inequality, and the celebrated Robertson-Schrödinger's VUR is based on the Cauchy-Schwarz inequality (and ensuing Parseval equality) [5]. Similarly, Fourier-type uncertainty relations are based on the Hausdorff-Young inequality [66], etc.

In information theory the key related inequalities are a) Young's inequality that implies the entropy power inequalities, b) the Riesz-Thorin inequality that determines the generalized entropic uncertainty relations and c) the Cramér-Rao and logarithmic Sobolev inequalities that imply Fisher's information uncertainty principle. In this section we will briefly review the concept of the entropy power and the ensuing entropy power inequality. Both concepts were developed by Shannon in his seminal 1948 paper in order to bound the capacity of non-Gaussian additive noise channels [23]. The connection with quantum mechanics was established by Stam [67], Lieb [72] and others who used the entropy power inequality to prove standard VUR.

In the second part of this section we show how the entropy power can be extended into the RE setting. With the help of Young's inequality we find the corresponding generalized entropy power inequality. Related applications to quantum mechanics will be postponed to Section VIB.

\section{A. Entropy power inequality — Shannon entropy case}

Suppose that $\mathcal{X}$ is a random vector in $\mathbb{R}^{D}$ with the $\mathrm{PDF} \mathcal{F}$. The differential (or continuous) entropy $\mathcal{H}(\mathcal{X})$ of $\mathcal{X}$ is defined as

$$
\mathcal{H}(\mathcal{X})=\mathcal{I}_{1}(\mathcal{F})=-\int_{\mathbb{R}^{D}} \mathcal{F}(\mathbf{x}) \log _{2} \mathcal{F}(\mathbf{x}) d \mathbf{x} .
$$

The discrete version of (5) is nothing but the Shannon entropy [23], and in such a case it represents an average number of binary questions that are needed to reveal the value of $\mathcal{X}$. Actually, (5) is not a proper entropy but rather information gain [21, 22] as can be seen directly from (44) when the limit $\alpha \rightarrow 1$ is taken. We shall return to this point in Section 5 . The entropy power $N(\mathcal{X})$ of $\mathcal{X}$ is the unique number such that [23, 24]

$$
\mathcal{H}(\mathcal{X})=\mathcal{H}\left(\mathcal{X}_{G}\right)
$$

where $\mathcal{X}_{G}$ is a Gaussian random vector with zero mean and variance equal to $N(\mathcal{X})$, i.e., $\mathcal{X}_{G} \sim \mathcal{N}\left(\mathbf{0}, N(\mathcal{X}) \mathbb{1}_{D \times D}\right)$. Eq.(6) can be equivalently rewritten in the form

$$
\mathcal{H}(\mathcal{X})=\mathcal{H}\left(\sqrt{N(\mathcal{X})} \cdot \mathcal{Z}_{G}\right)
$$


with $\mathcal{Z}_{G}$ representing a Gaussian random vector with the zero mean and unit covariance matrix. The solution of both (6) and (7) is then

$$
N(\mathcal{X})=\frac{2^{\frac{2}{D}} \mathcal{H}(\mathcal{X})}{2 \pi e}
$$

Let $\mathcal{X}_{1}$ and $\mathcal{X}_{2}$ be two independent continuous vector valued random variables of finite variance. In the case when the Shannon differential entropy is measured in nats (and not bits) we get for the entropy power

$$
N(\mathcal{X})=\frac{1}{2 \pi e} \exp \left(\frac{2}{D} \mathcal{H}(\mathcal{X})\right) .
$$

The differential entropy (8) (as well as (94)) satisfies the so-called entropy power inequality

$$
N\left(\mathcal{X}_{1}+\mathcal{X}_{2}\right) \geq N\left(\mathcal{X}_{1}\right)+N\left(\mathcal{X}_{1}\right)
$$

where the equality holds iff $\mathcal{X}_{1}$ and $\mathcal{X}_{2}$ are multivariate normal random variables with proportional covariance matrices [23]. In general, inequality (10) does not hold when $\mathcal{X}_{1}$ and $\mathcal{X}_{2}$ are discrete random variables and the differential entropy is replaced with the discrete entropy. Shannon originally used this inequality to obtain a lower bound for the capacity of non-Gaussian additive noise channels. Since Shannon's pioneering paper several proofs of the entropy power inequality have become available [54, 55, 57, 67].

\section{B. Entropy power inequality — Rényi entropy case}

In the following we will show how it is possible to extend the entropy power concept to REs. To this end we first define Rényi entropy power (for simplicity we use nats as units of information).

Definion III.1 Let $p>1$ and let $\mathcal{X}$ be a random vector in $\mathbb{R}^{D}$ with probability density $\mathcal{F} \in \ell^{p}\left(\mathbb{R}^{D}\right)$. The $p$-th Rényi entropy power of $\mathcal{X}$ is defined as

$$
N_{p}(\mathcal{X})=\frac{1}{2 \pi} p^{-p^{\prime} / p}\|\mathcal{F}\|_{p}^{-2 p^{\prime} / D}=\frac{1}{2 \pi} p^{-p^{\prime} / p} \exp \left(\frac{2}{D} \mathcal{I}_{p}(\mathcal{F})\right)
$$

where $p^{\prime}$ is the Hölder conjugate of $p$.

The above form of $N_{p}(\mathcal{X})$ was probably firstly stated by Gardner [58] who, however, did not develop the analogy with $N(\mathcal{X})$ any further. Plausibility of $N_{p}(\mathcal{X})$ as the entropy power comes from the following important properties:

Theorem III.1 The $p$-th Rényi entropy power $N_{p}(\mathcal{X})$ is a unique solution of the equation

$$
\mathcal{I}_{p}(\mathcal{X})=\mathcal{I}_{p}\left(\sqrt{N_{p}(\mathcal{X})} \cdot \mathcal{Z}_{G}\right)
$$


With $\mathcal{Z}_{G}$ representing a Gaussian random vector with zero mean and unit covariance matrix. In addition, in the limit $p \rightarrow 1_{+}$one has $N_{p}(\mathcal{X}) \rightarrow N(\mathcal{X})$.

Let $\mathcal{X}_{1}$ and $\mathcal{X}_{2}$ be two independent continuous random vectors in $\mathbb{R}^{D}$ with probability densities $\mathcal{F}^{(1)} \in \ell^{q}\left(\mathbb{R}^{D}\right)$ and $\mathcal{F}^{(2)} \in \ell^{p}\left(\mathbb{R}^{D}\right)$, respectively. Suppose further that $\lambda \in(0,1)$ and $r>1$, and let

$$
q=\frac{r}{(1-\lambda)+\lambda r}, \quad p=\frac{r}{\lambda+(1-\lambda) r}
$$

Then the following inequality holds:

$$
N_{r}\left(\mathcal{X}_{1}+\mathcal{X}_{2}\right) \geq\left(\frac{N_{q}\left(\mathcal{X}_{1}\right)}{1-\lambda}\right)^{1-\lambda}\left(\frac{N_{p}\left(\mathcal{X}_{2}\right)}{\lambda}\right)^{\lambda}
$$

Additionally, in the limits $r, p, q \rightarrow 1_{+}$the inequality (13) reduces to the Shannon entropy power inequality (10) and $N_{1}(\mathcal{X})=N(\mathcal{X})$.

Proof of Theorem III.1.

That $N_{p}(\mathcal{X})$ from Definition III.1 is the only solution of (12) follows from the scaling property of $\mathcal{I}_{p}$, namely

$$
\mathcal{I}_{p}(a \mathcal{X})=\mathcal{I}_{p}(\mathcal{X})+D \log _{2}|a|
$$

where $a \in \mathbb{R}$. The above scaling relation follows directly from the definition of $\mathcal{I}_{p}$ and from a change of variable argument. We can further use the simple fact that

$$
\mathcal{I}_{p}\left(\mathcal{Z}_{G}\right)=\frac{D}{2} \log _{2}\left(2 \pi p^{p^{\prime} / p}\right)
$$

to see that (12) leads to the equation

$$
\mathcal{I}_{p}(\mathcal{X})=\frac{D}{2} \log _{2}\left(2 \pi p^{p^{\prime} / p} N_{p}(\mathcal{X})\right)
$$

This yields

$$
N_{p}(\mathcal{X})=\frac{1}{2 \pi} p^{-p^{\prime} / p} 2^{\frac{2}{D} \mathcal{I}_{p}(\mathcal{X})}
$$

which, for $\mathcal{I}_{p}$ measured in nats, coincides with (11).

To prove the inequality (13) we first realize that $p, q$ and $r$ represent Hölder's triple, i.e.

$$
\frac{1}{q}+\frac{1}{p}=1+\frac{1}{r}
$$

This allows us to use Young's inequality (q.v. Appendix A), which for the case at hand reads

$$
\left\|\mathcal{F}^{(1)} * \mathcal{F}^{(2)}\right\|_{r} \leq C^{D}\left\|\mathcal{F}^{(1)}\right\|_{q}\left\|\mathcal{F}^{(2)}\right\|_{p}
$$


where $C$ is a constant defined in Appendix A. The left-hand-side of (19) can be explicitly written as

$$
\left\|\mathcal{F}^{(1)} * \mathcal{F}^{(2)}\right\|_{r}=\left[\int_{\mathbb{R}^{D}} d \mathbf{x}\left(\int_{\mathbb{R}^{D}} d \mathbf{y} \mathcal{F}^{(1)}(\mathbf{x}-\mathbf{y}) \mathcal{F}^{(2)}(\mathbf{y})\right)^{r}\right]^{1 / r} .
$$

The probability $\mathcal{F}^{(1)}(\mathbf{x}-\mathbf{y}) \mathcal{F}^{(2)}(\mathbf{y})$ is nothing but the joint probability that $\mathcal{X}_{1}=\mathbf{x}-\mathbf{y}$ and $\mathcal{X}_{2}=\mathbf{y}$. The quantity inside (...) thus represents the density function for the sum of two random variables $\mathcal{X}_{1}+\mathcal{X}_{2}=\mathbf{x}$. With the help of (11) we can rewrite (20) as

$$
\left\|\mathcal{F}^{(1)} * \mathcal{F}^{(2)}\right\|_{r}=\left[2 \pi N_{r}\left(\mathcal{X}_{1}+\mathcal{X}_{2}\right)\right]^{-D / 2 r^{\prime}} r^{-D / 2 r}
$$

On the other hand, the right-hand-side of (19) is

$$
\left\|\mathcal{F}^{(1)}\right\|_{q}\left\|\mathcal{F}^{(2)}\right\|_{p}=\left[2 \pi N_{q}\left(\mathcal{X}_{1}\right)\right]^{-D / 2 q^{\prime}}\left[2 \pi N_{p}\left(\mathcal{X}_{2}\right)\right]^{-D / 2 p^{\prime}} q^{-D / 2 q} p^{-D / 2 p}
$$

Plugging (21) and (22) into the Young inequality (19) we obtain

$$
\begin{aligned}
N_{r}\left(\mathcal{X}_{1}+\mathcal{X}_{2}\right) & \geq\left|r^{\prime}\right|^{-1}\left|q^{\prime}\right|^{-r^{\prime} / q^{\prime}}\left|p^{\prime}\right|^{-r^{\prime} / p^{\prime}}\left[N_{q}\left(\mathcal{X}_{1}\right)\right]^{r^{\prime} / q^{\prime}}\left[N_{p}\left(\mathcal{X}_{2}\right)\right]^{r^{\prime} / p^{\prime}} \\
& =\left(\frac{N_{q}\left(\mathcal{X}_{1}\right)}{1-\lambda}\right)^{1-\lambda}\left(\frac{N_{p}\left(\mathcal{X}_{2}\right)}{\lambda}\right)^{\lambda} .
\end{aligned}
$$

This completes the proof of the inequality (13).

It remains to show that in the limits $r, p, q \rightarrow 1_{+}$we regain the Shannon entropy power inequality. Firstly, the above limits directly give the inequality

$$
N\left(\mathcal{X}_{1}+\mathcal{X}_{2}\right) \geq\left(\frac{N\left(\mathcal{X}_{1}\right)}{1-\lambda}\right)^{1-\lambda}\left(\frac{N\left(\mathcal{X}_{2}\right)}{\lambda}\right)^{\lambda}
$$

which holds without restrictions on $\lambda \in(0,1)$. The best estimate (the highest lower bound) is obtained for $\lambda$ that extremizes the right-hand-side. Assuming that the right-hand-side is for fixed $\mathcal{X}_{1}$ and $\mathcal{X}_{2}$ a smooth function of $\lambda$, we can take its derivative with respect to $\lambda$. This equals zero when

$$
N\left(\mathcal{X}_{1}\right)=\left(\frac{1-\lambda}{\lambda}\right) N\left(\mathcal{X}_{2}\right) \quad \Leftrightarrow \quad \lambda=\frac{N\left(\mathcal{X}_{2}\right)}{N\left(\mathcal{X}_{1}\right)+N\left(\mathcal{X}_{2}\right)} .
$$

Positivity of $N(\ldots)$ then ensures that $\lambda$, which extremizes the right-hand-side of (24), belongs to the interval $(0,1)$. In addition, the extremum is actually a maximum because the second derivative is $-\left[N\left(\mathcal{X}_{1}\right)+N\left(\mathcal{X}_{2}\right)\right]^{3} / N\left(\mathcal{X}_{1}\right) N\left(\mathcal{X}_{2}\right)$ which is clearly negative. By inserting (25) into (24) we regain the Shannon entropy power inequality.

To prove that $N(\mathcal{X})$ is a limiting case of $N_{p}(\mathcal{X})$ for $p \rightarrow 1_{+}$, we just realize that $p^{-p^{\prime} / p} \rightarrow 1 / e$ and $\|\mathcal{F}\|_{p}^{-2 p^{\prime} / D} \rightarrow \exp \left(\frac{2}{D} \mathcal{I}_{1}(\mathcal{F})\right)$. Thus indeed in the $p \rightarrow 1_{+}$limit we regain the original Shannon entropy power $N(\mathcal{X})$ as well as the usual entropy power inequality (10).

In passing we may observe that from the definition (11) and Eqs. (14)-(15) it follows that $N_{p}\left(\sigma \mathcal{Z}_{G}\right)=\sigma^{2}$, i.e. the power entropy coincides for Gaussian processes with the variance $\sigma^{2}$. In case when $\mathcal{Z}_{G}$ represents a random Gaussian vector of zero mean and covariance matrix $\mathbf{K}$, then $N_{p}\left(\mathcal{Z}_{G}\right)=|\mathbf{K}|^{1 / D}$. Note that these statements are $p$-independent and hence valid also for the original Shannon entropy power. 


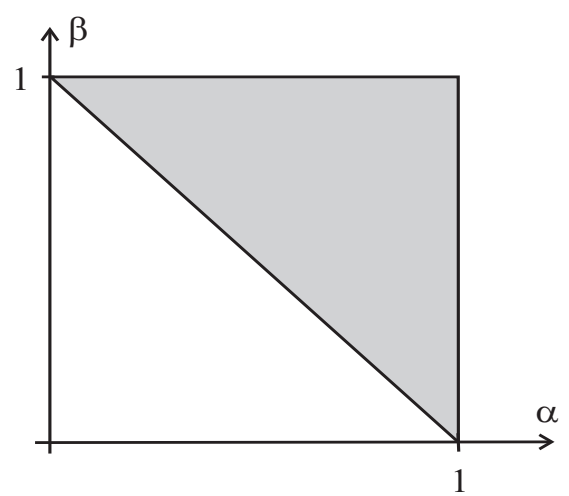

FIG. 1: Riesz convexity triangle. Riesz's inequality in Theorem 1 holds only when $\alpha$ and $\beta$ belong to the shaded region.

\section{INFORMATION THEORETIC UNCERTAINTY RELATIONS AND RÉNYI ENTROPY - DISCRETE DISTRIBUTIONS}

\section{A. The Riesz-Thorin Inequality}

To prove the information uncertainty relation based on RE we need to prove a particular variant of the Riesz-Thorin inequality [52, 53, 61] upon which our considerations will be based. For this purpose we first state the Riesz convexity theorem.

Theorem IV.1 (Riesz convexity theorem) Let $\mathcal{L}$ be a linear operator (i.e., $(\mathcal{L} \mathbf{x})_{j}=$ $\left.\sum_{i} a_{i j} x_{j}\right)$ and $\|\mathbf{y}\|_{p}=\left(\sum_{i}\left|y_{i}\right|^{p}\right)^{1 / p}$. Let, in addition, $M_{\alpha \beta}$ be the least number " $k$ " satisfying

$$
\|\mathcal{L} \mathbf{x}\|_{1 /(1-\beta)} \leq k\|\mathbf{x}\|_{1 / \alpha}
$$

Then $\log \left(M_{\alpha \beta}\right)$ is convex in triangle $0 \leq \alpha ; \beta \leq 1, \alpha+\beta \geq 1$.

The convexity triangle is depicted in Figure 1, Detailed exposition of the proof can be found for example in [61].

Corollary IV.1 Let $\left(\alpha_{1}, \beta_{1}\right)$ and $\left(\alpha_{2}, \beta_{2}\right)$ be two points in the above convex triangle. If we define

$$
\alpha=\alpha_{1} s+\alpha_{2}(1-s), \quad \beta=\beta_{1} s+\beta_{2}(1-s) ; \quad s \in[0,1]
$$

then clearly

$$
\log \left(M_{\alpha \beta}\right) \leq s \log \left(M_{\alpha_{1} \beta_{1}}\right)+(1-s) \log \left(M_{\alpha_{2} \beta_{2}}\right)
$$

or equivalently

$$
M_{\alpha \beta} \leq M_{\alpha_{1} \beta_{1}}^{s} M_{\alpha_{2} \beta_{2}}^{(1-s)}
$$


Theorem IV.2 (Riesz-Thorin inequality) Suppose that $(\mathcal{L} \mathbf{x})_{j}=\sum_{i} a_{j i} x_{i}$ and that

$$
\sum_{j}\left|(\mathcal{L} \mathbf{x})_{j}\right|^{2} \leq \sum_{j}\left|x_{j}\right|^{2}
$$

Then for $p \in[1,2]$ and $c \equiv \max _{i, j}\left|a_{i j}\right|$

$$
\|\mathcal{L} \mathbf{x}\|_{p^{\prime}} \leq c^{(2-p) / p}\|\mathbf{x}\|_{p}=c^{1 / p} c^{-1 / p^{\prime}}\|\mathbf{x}\|_{p} \quad \Leftrightarrow \quad c^{1 / p^{\prime}}\|\mathcal{L} \mathbf{x}\|_{p^{\prime}} \leq c^{1 / p}\|\mathbf{x}\|_{p},
$$

holds. Here $p$ and $p^{\prime}$ are Hölder conjugates, i.e., $1 / p+1 / p^{\prime}=1$.

Proof of Theorem IV.2. We shall use the notation $\alpha=1 / p, \beta=1 / q$ (and the Hölder conjugates $\left.p^{\prime}=p /(p-1), q^{\prime}=q /(q-1)\right)$. Consider the line from $\left(\alpha_{1}, \beta_{1}\right)=(1 / 2,1 / 2)$ to $\left(\alpha_{2}, \beta_{2}\right)=(1,1)$ in the $(\alpha, \beta)$ plane. This line lies entirely in the triangle of concavity (see Figure (1). Let us now define

$$
\begin{aligned}
\alpha & =\alpha_{1} s+\alpha_{2}(1-s) \\
& =s / 2+(1-s) \\
& =-s / 2+1,
\end{aligned}
$$

implying $s=2(1-\alpha)$, and define

$$
\begin{aligned}
\beta & =\beta_{1} s+\beta_{2}(1-s) \\
& =-s / 2+1,
\end{aligned}
$$

implying $\beta=\alpha$. Hence

$$
M_{\alpha, \alpha} \leq M_{\alpha_{1} \beta_{1}}^{s} M_{\alpha_{2} \beta_{2}}^{(1-s)}=M_{1 / 2,1 / 2}^{2(1-\alpha)} M_{1,1}^{2 \alpha-1} .
$$

Note particularly that because $s \in[0,1]$ then $\alpha \in[1 / 2,1]$ and $p \in[1,2]$. To estimate the right hand side of (26) we first realize that $M_{1 / 2,1 / 2} \leq 1$. This results from the very assumption of the theorem, namely that

$$
\|\mathcal{L} \mathbf{x}\|_{2}^{2}=\sum_{j}\left|(\mathcal{L} \mathbf{x})_{j}\right|^{2} \leq \sum_{j}\left|x_{j}\right|^{2}=\|\mathbf{x}\|_{2}^{2} .
$$

Hence, $M_{1 / 2,1 / 2} \leq k=1$. To find the estimate for $M_{11}$ we realize that it represents the smallest $k$ in the relation

$$
\|\mathcal{L} \mathbf{x}\|_{\infty} \leq k\|\mathbf{x}\|_{1}
$$

Thus

$$
M_{11}=\max _{\mathbf{x} \neq 0} \frac{\|\mathcal{L} \mathbf{x}\|_{\infty}}{\|\mathbf{x}\|_{1}}=\max _{\mathbf{x} \neq 0} \frac{\max _{j}\left|(\mathcal{L} \mathbf{x})_{j}\right|}{\sum_{i}\left|x_{i}\right|} \leq \max _{i, j}\left|a_{i j}\right| \equiv c .
$$

So finally we can write that

$$
M_{\alpha, \alpha}=M_{1 / p,\left(1-1 / p^{\prime}\right)} \leq c^{2 \alpha-1}=c^{(2-p) / p}=c^{1 / p} c^{-1 / p^{\prime}} .
$$




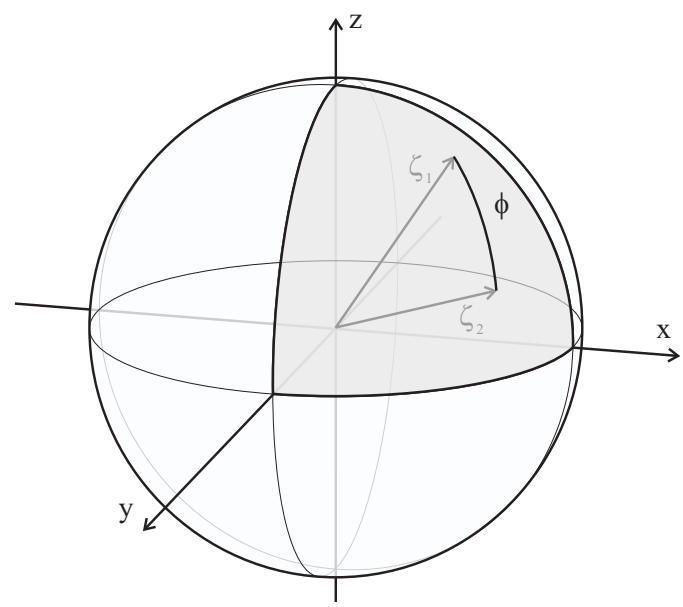

FIG. 2: A statistical system can be represented by points $\xi$ on a positive orthant $\left(S^{n-1}\right)^{+}$of the unit sphere $S^{n-1}$ in a real Hilbert space $H$. The depicted example corresponds to $n=3$.

\section{B. Generalized ITUR}

To establish the connection with $\mathrm{RE}$ let us assume that $\mathcal{X}$ is a discrete random variable with $n$ different values, $\mathbb{P}_{n}$ is the probability space affiliated with $\mathcal{X}$ and $\mathcal{P}=\left\{p_{1}, \ldots, p_{n}\right\}$ is a sample probability distribution from $\mathbb{P}_{n}$. Normally the geometry of $\mathbb{P}_{n}$ is identified with the geometry of a simplex. For our purpose it is more interesting to embed $\mathbb{P}_{n}$ in a sphere. Because $\mathcal{P}$ is non-negative and summable to unity, it follows that the square-root likelihood $\xi_{i}=\sqrt{p_{i}}$ exists for all $i=1, \ldots, n$, and it satisfies the normalization condition

$$
\sum_{i=1}^{n}\left(\xi_{i}\right)^{2}=1
$$

Hence $\xi$ can be regarded as a unit vector in the Hilbert space $\mathcal{H}=\mathbb{R}^{n}$. Then the inner product

$$
\cos \phi=\sum_{i=1}^{n} \xi_{i}^{(1)} \xi_{i}^{(2)}=1-\frac{1}{2} \sum_{i=1}^{n}\left(\xi_{i}^{(1)}-\xi_{i}^{(2)}\right)^{2},
$$

defines the angle $\phi$ that can be interpreted as a distance between two probability distributions. More precisely, if $\mathcal{S}^{n-1}$ is the unit sphere in the $n$-dimensional Hilbert space, then $\phi$ is the spherical (or geodesic) distance between the points on $\mathcal{S}^{n-1}$ determined by $\xi^{(1)}$ and $\xi^{(2)}$. Clearly, the maximal possible distance, corresponding to orthogonal distributions, is given by $\phi=\pi / 2$. This follows from the fact that $\xi^{(1)}$ and $\xi^{(2)}$ are non-negative, and hence they are located only on the positive orthant of $\mathcal{S}^{n-1}$ (see Figure 2). The geodesic distance $\phi$ is called the Bhattacharyya distance. The representation of probability distributions as points on a sphere also has an interesting relation to Bayesian statistics. If we use a uniform distribution on the sphere as the prior distribution then the prior distribution on probability vectors in $\mathbb{P}_{n}$ is exactly the celebrated Jeffrey's prior that has found new justification via the minimum description length approach to statistics [70]. 
Now, let $\mathcal{P}^{(1)}$ and $\mathcal{P}^{(2)}$ denote a pair of probability distributions and $\xi^{(1)}$ and $\xi^{(2)}$ the corresponding elements in Hilbert space. Because $\xi^{(1)}$ and $\xi^{(2)}$ are non-negative, they are located only on the positive orthant of $\mathcal{S}^{n-1}$. The transformation $\mathcal{L} \xi^{(1)}=\xi^{2}$ then corresponds to a rotation with $a_{i j} \in S O(n)$.

To proceed, we set $p^{\prime}=2(1+t)$ and $p=2(1+r)$ (remembering that $\left.1 / p+1 / p^{\prime}=1\right)$. Then the Riesz-Thorin inequality reads (with $\xi_{i}^{(1)} \leftrightarrow x_{i}$ )

$$
\left(\sum_{i}\left(\xi_{i}^{(2)}\right)^{p^{\prime}}\right)^{1 / p^{\prime}} \leq c^{(2-p) / p}\left(\sum_{i}\left(\xi_{i}^{(1)}\right)^{p}\right)^{1 / p}
$$

which is equivalent to

$$
\left(\sum_{j}\left(p_{j}^{(2)}\right)^{(1+t)}\right)^{1 / 2(1+t)}\left(\sum_{k}\left(p_{k}^{(1)}\right)^{(1+r)}\right)^{-1 / 2(1+r)} \leq c^{-r /(1+r)} .
$$

We raise both sides to the power $2(1+t) / t$ and get

$$
\left(\sum_{j}\left(p_{j}^{(2)}\right)^{(1+t)}\right)^{1 / t}\left(\sum_{k}\left(p_{k}^{(1)}\right)^{(1+r)}\right)^{-(1+t) / t(1+r)} \leq c^{-2 r(1+t) / t(1+r)} .
$$

The parameters are limited due to the condition $p \in[1,2]$ and $1 / p+1 / p^{\prime}=1$ implying

$$
t=-r /(2 r+1)
$$

This implies that $r \in[-1 / 2,0]$ and $t \in[0, \infty)$. Combining (30) and (31) we get

$$
\left(\sum_{j}\left(p_{j}^{(2)}\right)^{(1+t)}\right)^{1 / t}\left(\sum_{k}\left(p_{k}^{(1)}\right)^{(1+r)}\right)^{1 / r} \leq c^{2} .
$$

By applying the negative binary logarithm on both sides of (32) we get the following theorem.

Theorem IV.3 Suppose that $(\mathcal{L} \mathbf{x})_{j}=\sum_{i} a_{i j} x_{j} \equiv(\mathbb{A x})_{j}$ and that

$$
\sum_{j}\left|(\mathcal{L} \mathbf{x})_{j}\right|^{2} \leq \sum_{j}\left|x_{i}\right|^{2}, \quad \text { for all } x_{i}
$$

Define $c \equiv \max _{i, j}\left|a_{i j}\right|$. If $r \in[-1 / 2,0]$ and $t=-r /(2 r+1)$ and the probability distributions $\mathcal{P}^{(1)}$ and $\mathcal{P}^{(2)}$ are related by $\mathcal{L} \xi^{(1)}=\xi^{(2)}$ where $\xi_{i}=\sqrt{p_{i}}$, then

$$
\mathcal{I}_{1+t}\left(\mathcal{P}^{(2)}\right)+\mathcal{I}_{1+r}\left(\mathcal{P}^{(1)}\right) \geq-2 \log _{2} c .
$$

Two immediate comments are in order. Firstly, one can extend the domain of validity of both $r$ and $t$ by noticing that $\mathcal{P}^{(1)}$ and $\mathcal{P}^{(2)}$ are interchangeable in the above derivation without altering [60] the actual value of $c$. This has the consequence that one may phrase both resultant inequalities as a single inequality where both $r$ and $t$ belong to the interval $[-1 / 2, \infty)$ with $t=-r /(2 r+1)$. Secondly, because the information measure $\mathcal{I}_{\alpha}(\mathcal{P})$ is 
always non-negative, the inequality (33) can represent a genuine uncertainty relation only when $c<1$. Note that for $\mathbb{A} \in S O(n)$ or $S U(n)$ (i.e. for most physically relevant situations) one always has that $c \leq 1$. This is because for such $\mathbb{A}$ 's

$$
c=\max _{i, j}\left|a_{i k}\right|=\|\mathbb{A}\|_{\max } \leq\|\mathbb{A}\|_{2}=\sqrt{\lambda_{\max }\left(\mathbb{A}^{\dagger} \mathbb{A}\right)}=1 .
$$

The last identity results from the fact that all of eigenvalues of $\mathbb{A} \in S O(n)$ or $S U(n)$ have absolute value 1 .

It needs to be stressed that in the particular case when $r=0$ (and thus also $t=0$ ) we get

$$
\mathcal{H}\left(\mathcal{P}^{(2)}\right)+\mathcal{H}\left(\mathcal{P}^{(1)}\right) \geq-2 \log _{2} c .
$$

This Shannon entropy based uncertainty relation was originally found by Kraus 71] and Maassen [9]. A weaker version of this ITUR was also earlier proposed by Deutsch [8].

The reader can see that ITUR (33) which is based on RE provides a natural extension of the Shannon ITUR (35). In Section VI we shall see that there are quantum mechanical systems where Rényi's ITUR improves both on Robertson-Schrödinger's VUR and Shannon's ITUR.

\section{Geometric interpretation of inequality (33)}

Let us close this section by providing a useful geometric understanding of the inequality (33). To this end we invoke two concepts known from error analysis. These are, the condition number and distance to singularity (see, e.g., Refs. [79, 80]).

The condition number $\kappa_{\alpha, \beta}(\mathbb{A})$ of the non-singular matrix $\mathbb{A}$ is defined as

$$
\kappa_{\alpha, \beta}(\mathbb{A})=\|\mathbb{A}\|_{\alpha, \beta}\left\|\mathbb{A}^{-1}\right\|_{\beta, \alpha},
$$

where, the corresponding (mixed) matrix-valued norm $\|\mathbb{A}\|_{\alpha, \beta}$ is defined as

$$
\|\mathbb{A}\|_{\alpha, \beta}=\max _{\mathbf{x} \neq 0} \frac{\|\mathbb{A} \mathbf{x}\|_{\beta}}{\|\mathbf{x}\|_{\alpha}} .
$$

So, in particular $M_{11}=c$ from (27) is nothing but $\|\mathbb{A}\|_{1, \infty}$. Note also that $\|\mathbb{A}\|_{\alpha, \alpha}=\|\mathbb{A}\|_{\alpha}$, which is the usual $\alpha$-matrix norm. Justification for calling $\kappa_{\alpha, \beta}$ a condition number comes from the following theorem:

Theorem IV.4 Let $\mathbb{A} \mathbf{x}=\mathbf{y}$ be a linear equation and let there be an error (or uncertainty) $\delta \mathbf{y}$ in representing the vector $\mathbf{y}$, and let $\hat{\mathbf{x}}=\mathbf{x}+\delta \mathbf{x}$ solve the new error-hindered equation $\mathbb{A} \hat{\mathbf{x}}=\mathbf{y}+\delta \mathbf{y}$. The relative disturbance in $\mathbf{x}$ in relation to $\delta \mathbf{y}$ fulfills

$$
\frac{\|\delta \mathbf{x}\|_{\alpha}}{\|\mathbf{x}\|_{\alpha}} \leq \kappa_{\alpha, \beta}(\mathbb{A}) \frac{\|\delta \mathbf{y}\|_{\beta}}{\|\mathbf{y}\|_{\beta}} .
$$


Proof of Theorem IV.4. The proof is rather simple. Using the fact that $\mathbb{A} \mathbf{x}=\mathbf{y}$ and $\mathbb{A} \hat{\mathbf{x}}=\mathbf{y}+\delta \mathbf{y}$ we obtain $\delta \mathbf{x}=\mathbb{A}^{-1} \delta \mathbf{y}$. Taking $\alpha$-norm on both sides we can write

$$
\|\delta \mathbf{x}\|_{\alpha}=\left\|\mathbb{A}^{-1} \delta \mathbf{y}\right\|_{\alpha} \leq\left\|\mathbb{A}^{-1}\right\|_{\beta, \alpha}\|\delta \mathbf{y}\|_{\beta}
$$

On the other hand, the $\beta$-norm of $\mathbb{A} \mathbf{x}=\mathbf{y}$ yields

$$
\|\mathbf{y}\|_{\beta}=\|\mathbb{A} \mathbf{x}\|_{\beta} \leq\|\mathbb{A}\|_{\alpha, \beta}\|\mathbf{x}\|_{\alpha} \quad \Leftrightarrow \quad \frac{1}{\|\mathbf{x}\|_{\alpha}} \leq \frac{\|\mathbb{A}\|_{\alpha, \beta}}{\|\mathbf{y}\|_{\beta}}
$$

Combining (39) with (40) we obtain (38).

From the previous theorem we see that $\kappa_{\alpha, \beta}(\mathbb{A})$ quantifies a stability of the linear equation $\mathbb{A} \mathbf{x}=\mathbf{y}$, or better the extent to which the relative error (uncertainty) in $\mathbf{y}$ influences the relative error in $\mathbf{x}$. A system described by $\mathbb{A}$ and $\mathbf{y}$ is stable if $\kappa_{\alpha, \beta}(\mathbb{A})$ is not too large (ideally close to one). It is worth of stressing that $\kappa_{\alpha, \beta}(\mathbb{A}) \geq 1$. The latter results from the fact that

$$
\|\mathbf{x}\|_{\beta}=\left\|\mathbb{A A}^{-1} \mathbf{x}\right\|_{\beta} \leq\left\|\mathbb{A}^{-1}\right\|_{\beta, \beta}\|\mathbf{x}\|_{\beta} \leq\|\mathbb{A}\|_{\alpha, \beta}\left\|\mathbb{A}^{-1}\right\|_{\beta, \alpha}\|\mathbf{x}\|_{\beta}
$$

In the last step we have used the submultiplicative property of mixed matrix norms.

The second concept — the distance to singularity for a matrix $\mathbb{A}$, is defined as

$$
\operatorname{dist}_{\alpha, \beta}(\mathbb{A}) \equiv \min \left\{\|\Delta \mathbb{A}\|_{\alpha, \beta} ; \mathbb{A}+\Delta \mathbb{A} \text { singular }\right\} \text {. }
$$

In this connection an important theorem states that the relative distance to singularity is the reciprocal of the condition number.

Theorem IV.5 For a non-singular matrix $\mathbb{A}$, one has

$$
\frac{\operatorname{dist}_{\alpha, \beta}(\mathbb{A})}{\|\mathbb{A}\|_{\alpha, \beta}}=\kappa_{\alpha, \beta}(\mathbb{A})^{-1} \text {. }
$$

Proof of Theorem IV.5. If $\mathbb{A}+\Delta \mathbb{A}$ is singular then there is a vector $\mathbf{x} \neq 0$, such that $(\mathbb{A}+\Delta \mathbb{A}) \mathbf{x}=0$. Because $\mathbb{A}$ is non-singular, the latter is equivalent to $\mathbf{x}=-\mathbb{A}^{-1} \Delta \mathbb{A} \mathbf{x}$. By taking the $\alpha$-norm we have

$$
\|\mathbf{x}\|_{\alpha}=\left\|\mathbb{A}^{-1} \Delta \mathbb{A} \mathbf{x}\right\|_{\alpha} \leq\left\|\mathbb{A}^{-1}\right\|_{\beta, \alpha}\|\Delta \mathbb{A} \mathbf{x}\|_{\beta} \leq\left\|\mathbb{A}^{-1}\right\|_{\beta, \alpha}\|\Delta \mathbb{A}\|_{\alpha, \beta}\|\mathbf{x}\|_{\alpha},
$$

which is equivalent to

$$
\frac{\|\Delta \mathbb{A}\|_{\alpha, \beta}}{\|\mathbb{A}\|_{\alpha, \beta}} \geq \kappa_{\alpha, \beta}(\mathbb{A})^{-1} .
$$

To show that $\kappa_{\alpha, \beta}(\mathbb{A})^{-1}$ is a true minimum of the left-hand side of (45) and not mere lower bound we must show that there exists such a suitable perturbation $\Delta \mathbb{A}$ which saturates the inequality. Corresponding $\|\Delta \mathbb{A}\|_{\alpha, \beta}$ will then clearly represent $\operatorname{dist}_{\alpha, \beta}(\mathbb{A})$. Consider y such 
that $\|\mathbf{y}\|_{\beta}=1$ and $\left\|\mathbb{A}^{-1} \mathbf{y}\right\|_{\alpha}=\left\|\mathbb{A}^{-1}\right\|_{\beta, \alpha}$, and write $\mathbf{z}=\mathbb{A}^{-1} \mathbf{y}$. Define further a vector $\hat{\mathbf{z}}$ such that

$$
\max _{\|\zeta\|_{\alpha}=1} \frac{\left|\hat{\mathbf{z}}^{*} \cdot \zeta\right|}{\|\mathbf{z}\|_{\alpha}}=\frac{\hat{\mathbf{z}}^{*} \cdot \mathbf{z}}{\|\mathbf{z}\|_{\alpha}}=1 .
$$

We now introduce the matrix $\mathbb{B}_{i, j}=-\mathbf{y}_{i} \hat{\mathbf{z}}_{j}^{*}$, which implies $\mathbb{B} \mathbf{z} /\|\mathbf{z}\|_{\alpha}=-\mathbf{y}$. Note that $\mathbb{B}$ thus defined fulfills

$$
\|\mathbb{B}\|_{\alpha, \beta}=\max _{\|\zeta\|_{\alpha}=1} \frac{\left\|\mathbf{y}\left(\hat{\mathbf{z}}^{*} \cdot \zeta\right)\right\|_{\beta}}{\|\mathbf{z}\|_{\alpha}}=\|\mathbf{y}\|_{\beta} \max _{\|\zeta\|_{\alpha}=1} \frac{\left|\hat{\mathbf{z}}^{*} \cdot \zeta\right|}{\|\mathbf{z}\|_{\alpha}}=1 .
$$

Let us set $\Delta \mathbb{A}=\mathbb{B} /\|\mathbf{z}\|_{\alpha}$. This directly implies that

$$
(\mathbb{A}+\Delta \mathbb{A}) \mathbb{A}^{-1} \mathbf{y}=\mathbf{y}+\frac{\mathbb{B} \mathbf{z}}{\|\mathbf{z}\|_{\alpha}}=0
$$

So the matrix $\mathbb{A}+\Delta \mathbb{A}$ is singular with $\mathbb{A}^{-1} \mathbf{y}$ being the null vector. Finally note that

$$
\frac{\|\Delta \mathbb{A}\|_{\alpha, \beta}}{\|\mathbb{A}\|_{\alpha, \beta}}=\frac{\|\mathbb{B}\|_{\alpha, \beta}}{\|\mathbf{z}\|_{\alpha}\|\mathbb{A}\|_{\alpha, \beta}}=\frac{1}{\left\|\mathbb{A}^{-1} \mathbf{y}\right\|_{\alpha}\|\mathbb{A}\|_{\alpha, \beta}}=\kappa_{\alpha, \beta}(\mathbb{A})^{-1} .
$$

The connection with the ITUR (33) is established when we observe that the smallest value of $c$ is (see, Eqs.(27) and (43))

$$
c=\|\mathbb{A}\|_{1, \infty}=\operatorname{dist}_{1, \infty}(\mathbb{A}) \kappa_{1, \infty}(\mathbb{A}) .
$$

Since $c \leq 1$, this shows that the ITUR (33) restricts the probability distributions more the smaller the distance to singularity and/or the lower the stability of the transformation matrix $\mathbb{A}$ is. In practical terms this means that the rotation/transformation within the positive orthant introduces higher ignorance or uncertainty in the ITUR the more singular the rotation/transformation matrices are.

\section{INFORMATION THEORETIC UNCERTAINTY RELATIONS AND RÉNYI ENTROPY - CONTINUOUS DISTRIBUTIONS}

Before considering quantum-mechanical implications of Rényi's ITUR (33), we will briefly touch upon the continuous-probability analogue of (33). This issue is conceptually far more delicate than the discrete one namely because it is difficult to find norms for the correspondent (integro-)differential operators $\mathcal{L}$. This in particular does not allow one to calculate explicitly the optimal bounds in many relevant cases. Fortunately, there is one very important class of situations, where one can proceed with relative ease. This is the situation when the linear transform is represented by a continuous Fourier transform, in which case the Riesz-Thorin inequality is taken over by the Beckner-Babebko inequality [62, 63].

\section{Theorem V.1 (Beckner-Babebko's theorem) Let}

$$
f^{(2)}(\mathbf{x}) \equiv \hat{f}^{(1)}(\mathbf{x})=\int_{\mathbb{R}^{D}} e^{2 \pi i \mathbf{x} \cdot \mathbf{y}} f^{(1)}(\mathbf{y}) d \mathbf{y},
$$


then for $p \in[1,2]$ we have

$$
\|\hat{f}\|_{p^{\prime}} \leq \frac{\left|p^{D / 2}\right|^{1 / p}}{\left|\left(p^{\prime}\right)^{D / 2}\right|^{1 / p^{\prime}}}\|f\|_{p}
$$

or, equivalently

$$
\left|\left(p^{\prime}\right)^{D / 2}\right|^{1 / p^{\prime}}\left\|f^{(2)}\right\|_{p^{\prime}} \leq\left|p^{D / 2}\right|^{1 / p}\left\|f^{(1)}\right\|_{p}
$$

Here, again, $p$ and $p^{\prime}$ are the usual Hölder conjugates. For any $F \in \ell^{p}\left(\mathbb{R}^{D}\right)$ the $p$-norm $\|F\|_{p}$ is defined as

$$
\|F\|_{p}=\left(\int_{\mathbb{R}^{D}}|F(\mathbf{y})|^{p} d \mathbf{y}\right)^{1 / p} .
$$

Due to symmetry of the Fourier transform the reverse inequality also holds:

$$
\|f\|_{p^{\prime}} \leq \frac{\left|p^{D / 2}\right|^{1 / p}}{\left|\left(p^{\prime}\right)^{D / 2}\right|^{1 / p^{\prime}}}\|\hat{f}\|_{p}
$$

The proof of this theorem can be found in the Appendix. Lieb [64] proved that the inequality (50) is saturated only for Gaussian functions. In the case of discrete Fourier transforms the corresponding inequality is known as the (classical) Hausdorff-Young inequality [61, 66].

Analogous manipulations that have brought us from equation (29) to equation (32) will allow us to cast (50) in the form

$$
\left(\int_{\mathbb{R}^{D}}\left[\mathcal{F}^{(2)}(\mathbf{y})\right]^{(1+t)} d \mathbf{y}\right)^{1 / t}\left(\int_{\mathbb{R}^{D}}\left[\mathcal{F}^{(1)}(\mathbf{y})\right]^{(1+r)} d \mathbf{y}\right)^{1 / r} \leq[2(1+t)]^{D}|t / r|^{D / 2 r}
$$

where we have defined the square-root density likelihood as $|f(\mathbf{y})|=\sqrt{\mathcal{F}(\mathbf{y})}$.

When the negative binary logarithm is applied to both sides of (52), then

$$
\mathcal{I}_{1+t}\left(\mathcal{F}^{(2)}\right)+\mathcal{I}_{1+r}\left(\mathcal{F}^{(1)}\right) \geq-D+\frac{1}{r} \log _{2}(1+r)^{D / 2}+\frac{1}{t} \log _{2}(1+t)^{D / 2} .
$$

Because $1 / t+1 / r=-2$, we can recast the previous inequality in the equivalent form

$$
\mathcal{I}_{1+t}\left(\mathcal{F}^{(2)}\right)+\mathcal{I}_{1+r}\left(\mathcal{F}^{(1)}\right) \geq \frac{1}{r} \log _{2}[2(1+r)]^{D / 2}+\frac{1}{t} \log _{2}[2(1+t)]^{D / 2} .
$$

This can be further simplified by looking at the minimal value of the RHS of (53) (or (54)) under the constraint $1 / t+1 / r=-2$. The minimal value is attained for $t=-1 / 2$ and equivalently $t=\infty$, see Fig. 3, and it is 0 (note $t$ cannot be smaller than $-1 / 2$ ). This in particular implies that

$$
\mathcal{I}_{1+t}\left(\mathcal{F}^{(2)}\right)+\mathcal{I}_{1+r}\left(\mathcal{F}^{(1)}\right) \geq 0
$$

Inequality (53) is naturally stronger than (55), but the latter is usually much easier to implement in practical calculations. In addition the RHS of (53) is universal in the sense that it is $t$ and $r$ independent. The reader should also notice that the zero value of the 


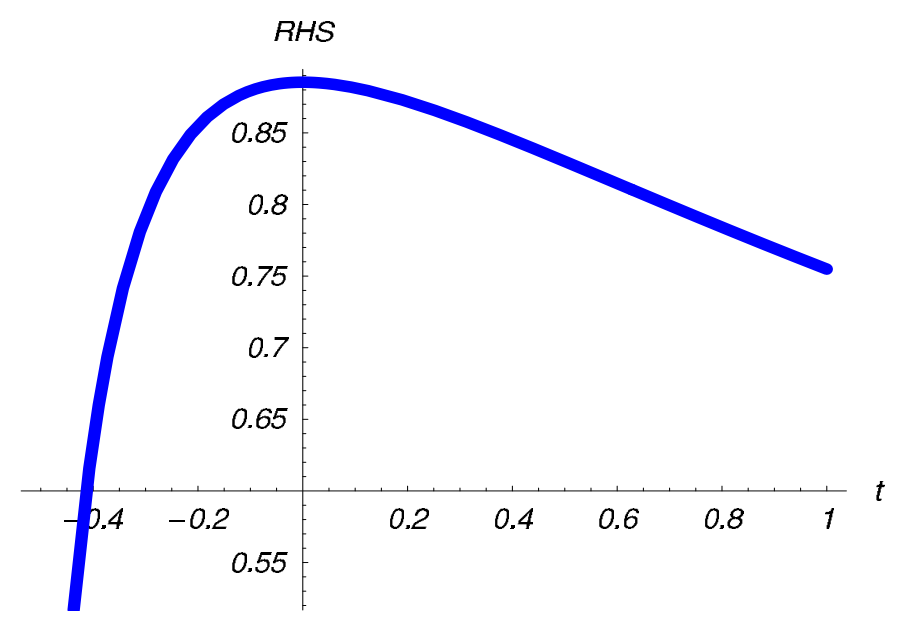

FIG. 3: Dependence of the RHS of (54) on $t$ provided we substitute for $r=-t /(2 t+1)$. The minimum is attained for $t=-1 / 2$ and $t=\infty$ (and hence $r=\infty$ and $r=-1 / 2$, respectively) while maximum is at $t=0_{+}$(and hence for $r=0_{-}$).

right-hand side of (55) does not yield a trivial inequality since $\mathcal{I}_{\alpha}$ are not generally positive for continuous PDFs (q.v. Section 4.2). In fact, from the coarse probability version of (55) (cf. Eq. (4) follows

$$
\mathcal{I}_{1+t}\left(\mathcal{P}_{n}^{(2)}\right)+\mathcal{I}_{1+r}\left(\mathcal{P}_{n}^{(1)}\right) \geq-2 D \log _{2} l,
$$

which is clearly a non-trivial ITUR.

Also, notice that in the limit $t \rightarrow 0_{+}$and $r \rightarrow 0_{-}$the inequalities (53)-(54) take the form [86]

$$
\mathcal{H}\left(\mathcal{F}^{(2)}\right)+\mathcal{H}\left(\mathcal{F}^{(1)}\right) \geq \log _{2}\left(\frac{e}{2}\right)^{D},
$$

which coincides with the classical Hirschman conjecture for the differential Shannon entropy based ITUR [81]. In this connection it should be noted that among all admissible pairs $\{r, t\}$, the pair $\left\{0_{-}, 0_{+}\right\}$gives the highest value of the RHS in (54). This can be clearly seen from Fig. 3 ,

Let us finally observe that when (53)-(54) is rewritten in the language of Rényi entropy powers it can be equivalently cast in the form

$$
N_{1+t}\left(\mathcal{F}^{(2)}\right) N_{1+r}\left(\mathcal{F}^{(1)}\right) \equiv N_{1+t}(\mathcal{X}) N_{1+r}(\mathcal{Y}) \geq \frac{1}{16 \pi^{2}},
$$

or equivalently as

$$
N_{p / 2}(\mathcal{X}) N_{q / 2}(\mathcal{Y}) \geq \frac{1}{16 \pi^{2}},
$$

with $p$ and $q$ being Hölder conjugates and the RE measured in bits. Note that in the case when both $\mathcal{X}$ and $\mathcal{Y}$ represent random Gaussian vectors then (59) reduces to

$$
\left|K_{\mathcal{X}}\right|^{1 / D}\left|K_{\mathcal{Y}}\right|^{1 / D}=\frac{1}{16 \pi^{2}} .
$$


Here, $\left|K_{\mathcal{X}}\right|$ and $\left|K_{\mathcal{Y}}\right|$ are determinants of the respective covariance matrices. The equality follows from the Lieb condition on the saturation of the Beckner-Babenko inequality. It is also interesting to notice that when we define the variance per component, i.e.,

$$
\begin{aligned}
\sigma_{\mathcal{X}}^{2} & =\operatorname{Var}(\mathcal{X}) / D=\operatorname{Tr}\left[\left(K_{\mathcal{X}}\right)_{i j}\right] / D, \\
\sigma_{\mathcal{Y}}^{2} & =\operatorname{Var}(\mathcal{Y}) / D=\operatorname{Tr}\left[\left(K_{\mathcal{Y}}\right)_{i j}\right] / D,
\end{aligned}
$$

then, from (60), these satisfy

$$
\sigma_{\mathcal{X}}^{2} \sigma_{\mathcal{Y}}^{2} \geq \frac{1}{16 \pi^{2}} .
$$

The proof is based on the identity

$$
\log (\operatorname{det} \mathbb{A})=\operatorname{Tr}(\log \mathbb{A}),
$$

which is certainly valid for any diagonalizable matrix $\mathbb{A}$, and more generally for all matrices since diagonalizable matrices are dense. With this we have

$$
\begin{aligned}
\log \left|K_{\mathcal{X}}\right|^{1 / D} & =\operatorname{Tr}\left[\frac{1}{D}\left(K_{\mathcal{X}}\right)_{i j}\right]=\sum_{i=1}^{D}\left[\frac{1}{D} \log \left(K_{\mathcal{X}}\right)_{i i}\right] \\
& \leq \log \left[\sum_{i=1}^{D} \frac{1}{D}\left(K_{\mathcal{X}}\right)_{i i}\right]=\log \left\{\operatorname{Tr}\left[\left(K_{\mathcal{X}}\right)_{i j}\right] / D\right\}=\log \sigma_{\mathcal{X}}^{2} .
\end{aligned}
$$

The inequality follows from Jensen's inequality for the logarithm. An analogous result holds also for the random vector $\mathcal{Y}$. The equality in (65) holds only when $\mathcal{X}$ is white, i.e., if its covariance matrix is proportional to the identity matrix. If the components of the random Gaussian vector are independent, it makes sense (in view of the additivity of the RE) to speak about the RE (and ensuing entropy power) of a given random component. In that case (60) boils down to

$$
\sigma_{\mathcal{X}_{i}}^{2} \sigma_{\mathcal{Y}_{i}}^{2}=\frac{1}{16 \pi^{2}},
$$

where the subscript $i$ denotes the $i$-th component of the random vector.

Inequalities (58)-(59) make the connection of the continuous ITUR with the VUR. This is because when the distributions in question have finite covariance matrices then the following theorem holds:

Theorem V.2 Let $\mathcal{X}$ be a random vector in $\mathbb{R}^{D}$ with the finite covariance matrix $\left(K_{\mathcal{X}}\right)_{i j}$. Then

$$
N(\mathcal{X}) \leq\left|K_{\mathcal{X}}\right|^{1 / D} \leq \sigma_{\mathcal{X}}^{2},
$$

with equality in the first inequality if and only if $\mathcal{X}$ is a Gaussian vector, and in the second if and only if $\mathcal{X}$ is white. 
The proof of this theorem is based on the non-negativity of the relative Shannon entropy (or Kullback-Leibler divergence) and can be found, e.g., in Refs. [68, 69]. An important upshot of the previous theorem is that also for non-Gaussian distributions one has

$$
\sigma_{\mathcal{X}}^{2} \sigma_{\mathcal{Y}}^{2} \geq\left|K_{\mathcal{X}}\right|^{1 / D}\left|K_{\mathcal{Y}}\right|^{1 / D} \geq N(\mathcal{X}) N(\mathcal{Y}) \geq \frac{1}{16 \pi^{2}}
$$

which saturates only for Gaussian (respective white) random vectors $\mathcal{X}$ and $\mathcal{Y}$. This is just one example where a well-known inequality can be improved by replacing variance by a quantity related to entropy. In certain cases the last inequality in (68) can be improved by using Rényi's entropy power rather than Shannon's entropy power. In fact, the inequality

$$
N(\mathcal{X}) N(\mathcal{Y}) \geq N_{p / 2}(\mathcal{X}) N_{q / 2}(\mathcal{Y}) \geq \frac{1}{16 \pi^{2}},
$$

(with $p$ and $q$ being Hölder conjugates) is fulfilled whenever

$$
\mathcal{H}(\mathcal{X})-\mathcal{I}_{p / 2}(\mathcal{X}) \geq \mathcal{I}_{q / 2}(\mathcal{Y})-\mathcal{H}(\mathcal{Y})
$$

$(q \in[1,2]$ and $p \in[2, \infty))$. Note that both sides in (70) are positive (cf. Eq. (44)). Inequality (701) can be satisfied by a number of PDFs. This is often the case when the PDF $\mathcal{F}^{(1)}$ associated with $\mathcal{Y}$ is substantially leptokurtic (peaked) while $\mathcal{F}^{(2)}$ (which is related to $\mathcal{X}$ ) is platykurtic heavy-tailed PDF. A simple example is the Cauchy-Lorentz distribution [11], for which we have

$$
\begin{aligned}
& f(x)=\sqrt{\frac{c}{\pi}} \sqrt{\frac{1}{c^{2}+x^{2}}}, \\
& \hat{f}(y)=\sqrt{\frac{2 c}{\pi^{2}}} K_{0}(c|y|), \\
& \mathcal{F}^{(2)}(x)=\frac{c}{\pi} \frac{1}{c^{2}+x^{2}}, \\
& \mathcal{F}^{(1)}(y)=\frac{2 c}{\pi^{2}} K_{0}^{2}(c|y|) .
\end{aligned}
$$

Here $\mathcal{F}^{(2)}(x)$ is the Cauchy-Lorentz PDF. In Fig 4 we graphically represent the LHS and the RHS of the inequality of (70) for PDFs (73)-(74). There we can see that the inequality becomes strongest if we choose $p \rightarrow \infty$ and $q=1$. On the basis of our numerical simulations it seems that the behavior depicted in Fig 4 is quite common for heavy-tailed Lévy stable distributions.

Of course, in cases when the covariance matrices are infinite, Theorem V.2 does not hold and the continuous ITUR remains the only sensible quantifier of the inherent QM uncertainty. We shall delve more into the quantum mechanics implications of the above (continuous PDF) ITUR inequalities in Section VIB,

\section{APPLICATIONS IN QUANTUM MECHANICS}

The connection of the information theoretic inequalities (33) and (53) with quantum

mechanics is established when we consider two quantum-mechanical observables, say $\hat{A}$ and 


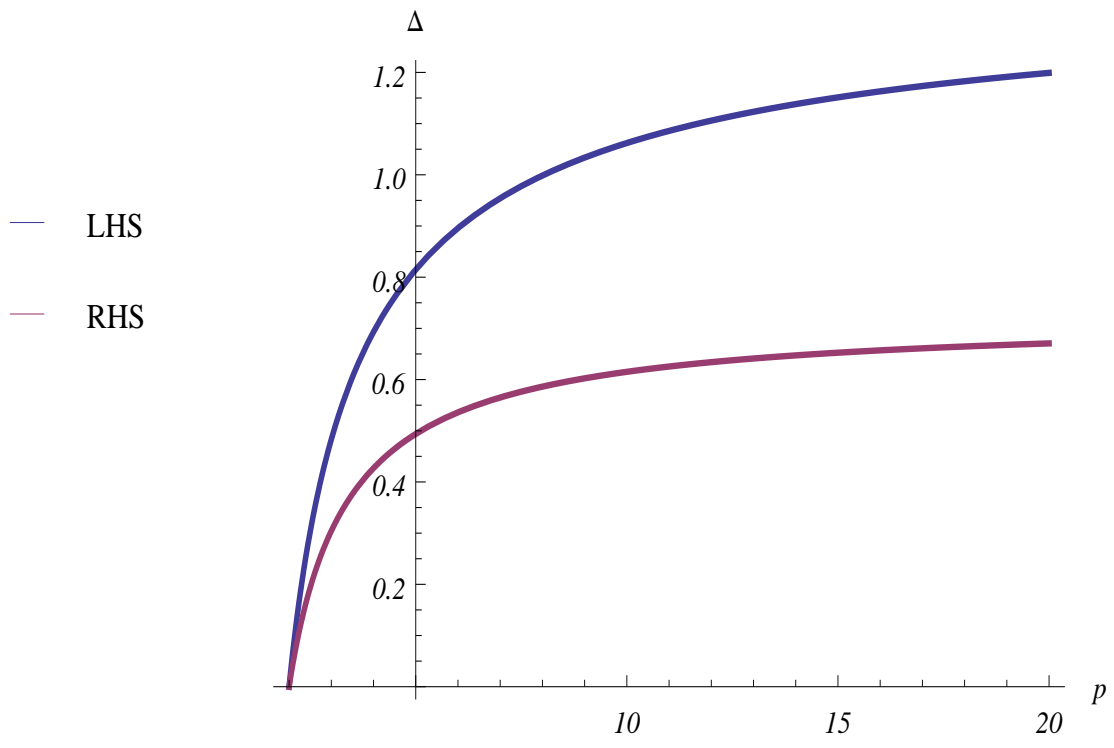

FIG. 4: Graphical representation of the LHS and the RHS of the inequality of (70). $\Delta$ denotes the difference of entropies on the LHS respective the RHS of (70). The $q$ variable is phrased in terms of $p$ as $q=p /(p-1)$. Because the PDF's involved have no fundamental scale, the ensuing entropies (and hence $\Delta$ ) are $c$ independent. Note in particular that the inequality (70) is strongest for $p \rightarrow \infty$ and $q=1$.

$\hat{B}$, written through their spectral decompositions

$$
\hat{A}=\sum a|a\rangle\left\langle a\left|d a, \quad \hat{B}=\sum b\right| b\right\rangle\langle b| d b .
$$

Here the integral-summation symbol schematically represents summation over a discrete part of the spectra and integration over a continuous part of the spectra. States $|a\rangle$ and $|b\rangle$ are proper (for discrete spectrum) and improper (for the continuous spectrum) eigenvectors of $\hat{A}$ and $\hat{B}$, respectively.

According to the quantum measurement postulate, the probability of obtaining a result $a$ in a measurement of observable $\hat{A}$ on a system prepared in the state $|\phi\rangle$ is given by the (transition) probability density

$$
\mathcal{F}(a)=|\langle a \mid \phi\rangle|^{2} .
$$

When $a_{i}$ belongs to a discrete spectrum, then the (transition) probability for the result $a_{i}$ is

$$
p\left(a_{i}\right)=\left|\left\langle a_{i} \mid \phi\right\rangle\right|^{2} .
$$

Similarly for the observable $\hat{B}$. 


\section{A. Discrete probabilities}

For the discrete-spectrum the conditions assumed in the Riesz-Thorin inequality (cf. Theorem IV.2) are clearly fulfilled by setting $x_{i}=\left\langle x_{i} \mid \phi\right\rangle,(\mathcal{L} \mathbf{x})_{j}=\left\langle b_{j} \mid \phi\right\rangle$ and $a_{i j}=\left\langle b_{j} \mid a_{k}\right\rangle$. We will now illustrate the utility of Rényi's ITUR with a toy-model example. To this end we consider a two-dimensional state $|\phi\rangle$ of a spin- $\frac{1}{2}$ particle, and let $\hat{A}$ and $\hat{B}$ be spin components in orthogonal directions, i.e.

$$
|A\rangle \equiv\left(\begin{array}{c}
\left|S_{x} ;+\right\rangle \\
\left|S_{x} ;-\right\rangle
\end{array}\right), \quad|B\rangle \equiv\left(\begin{array}{c}
\left|S_{z} ;+\right\rangle \\
\left|S_{z} ;-\right\rangle
\end{array}\right) .
$$

Because

$$
\left(\begin{array}{l}
\left|S_{x} ;+\right\rangle \\
\left|S_{x} ;-\right\rangle
\end{array}\right)=\frac{1}{\sqrt{2}}\left(\begin{array}{cc}
1 & 1 \\
-1 & 1
\end{array}\right)\left(\begin{array}{l}
\left|S_{z} ;+\right\rangle \\
\left|S_{z} ;-\right\rangle
\end{array}\right)
$$

we can immediately identify $c$ with $1 / \sqrt{2}$ (cf. Eq. (35)). Let us now define probability $\mathcal{P}=(p,(1-p)) \equiv\left(\left|\left\langle S_{x} ;+\mid \phi\right\rangle\right|^{2},\left|\left\langle S_{x} ;-\mid \phi\right\rangle\right|^{2}\right)$. Without loss of generality we may assume that $p=\max _{i} \mathcal{P}$. The question we are interested in is how the knowledge of $\mathcal{P}$ restricts the distribution $\mathcal{Q}=(q,(1-q)) \equiv\left(\left|\left\langle S_{z} ;+\mid \phi\right\rangle\right|^{2},\left|\left\langle S_{z} ;-\mid \phi\right\rangle\right|^{2}\right)$ ? Both distribution cannot be independent as Shannon's ITUR

$$
\mathcal{H}(\mathcal{P})+\mathcal{H}(\mathcal{Q}) \geq-2 \log _{2} c=1
$$

clearly indicates. In fact, inequality (80) can be equivalently phrased in the form

$$
p^{p}(1-p)^{1-p} \leq \frac{1}{2} q^{-q}(1-q)^{q-1} .
$$

The graphical solution of this equation can be seen on Fig. 5. In case of Rényi's ITUR we

can take advantage of the fact that the RE is a monotonically decreasing function of its index and hence the most stringent relation between $p$ and $q$ is provided via Rényi's ITUR

$$
\mathcal{I}_{\infty}(\mathcal{P})+\mathcal{I}_{1 / 2}(\mathcal{Q}) \geq-2 \log _{2} c=1
$$

The latter is equivalent to

$$
\sqrt{q} \sqrt{1-q}+1 / 2 \geq p
$$

This inequality can be again treated graphically, see Fig. 6. The comparison with the ordinary Schrödinger-Robertson's VUR, can easily be made. In fact, we have

$$
\left\langle\left(\triangle S_{x}\right)^{2}\right\rangle_{\phi}\left\langle\left(\triangle S_{z}\right)^{2}\right\rangle_{\phi} \geq \frac{\hbar^{2}}{4}\left|\left\langle S_{y}\right\rangle_{\phi}\right|^{2} \Leftrightarrow p(1-p) \geq \frac{1}{4} \sin ^{2}\left(\varphi_{+}-\varphi_{-}\right)
$$

where the phase $\varphi_{ \pm}$is defined as

$$
e^{i \varphi_{ \pm}} \equiv \frac{\left\langle\phi \mid S_{z} ; \pm\right\rangle}{\left|\left\langle\phi \mid S_{z} ; \pm\right\rangle\right|} .
$$



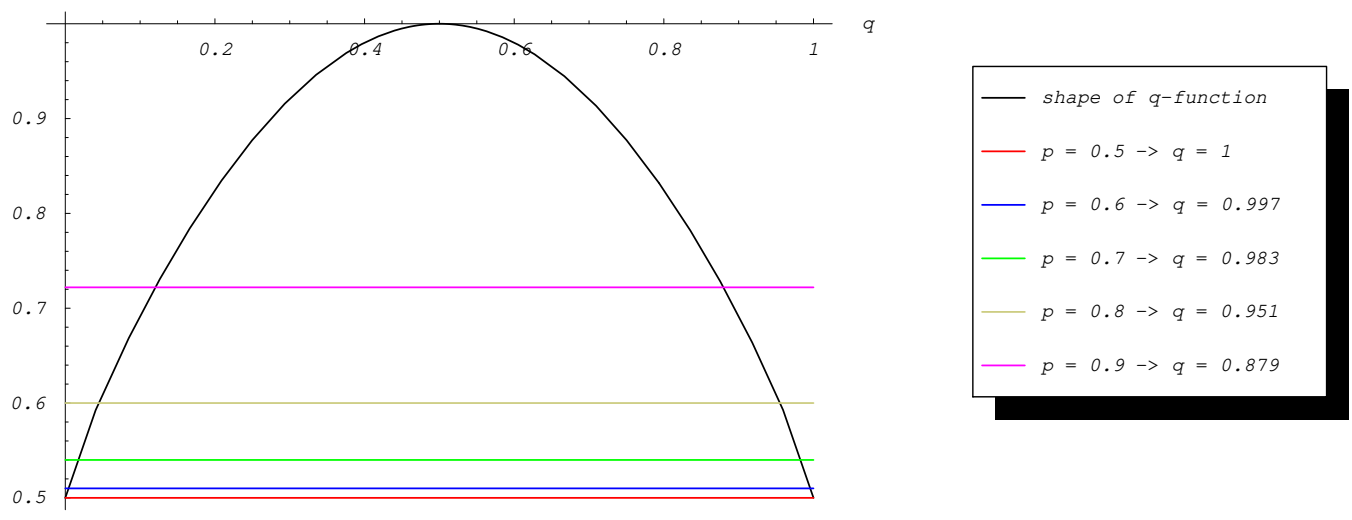

FIG. 5: Graphical representation of the inequality (81). For fixed value of $p$ the inequality is fulfilled for all $q$ 's that lie inside the $q$-function, i.e., function $y=\frac{1}{2} q^{-q}(1-q)^{q-1}$.
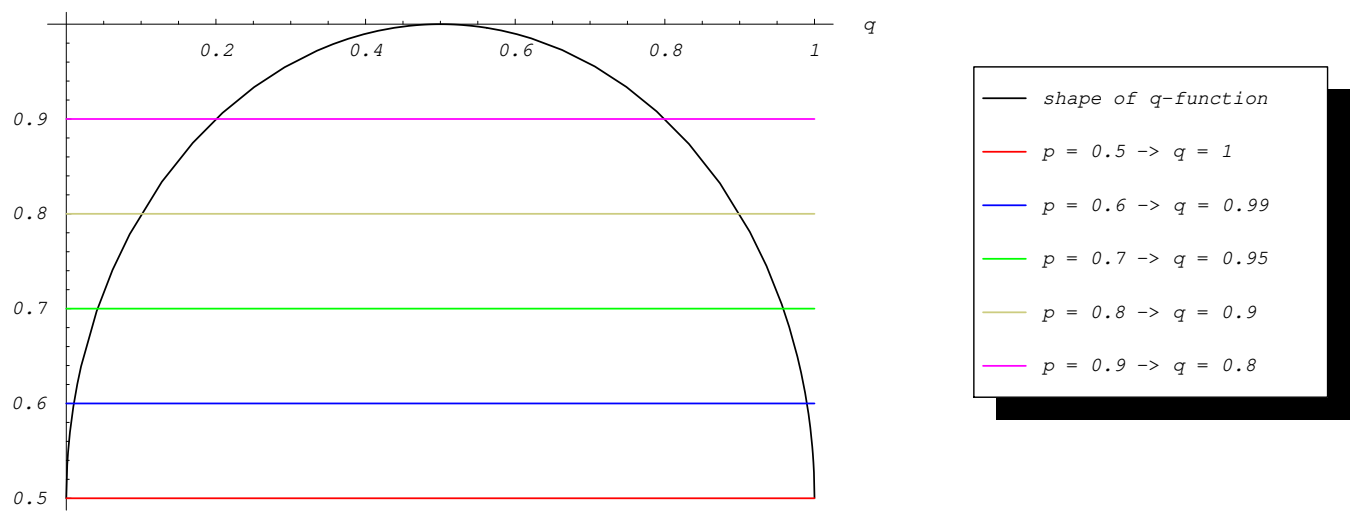

FIG. 6: Graphical representation of the inequality (83). For fixed value of $p$ the inequality is fulfilled for all $q$ 's that lie inside the $q$-function, i.e., function $y=\sqrt{q} \sqrt{1-q}+1 / 2$.

In deriving this we have used the relation

$$
\left(\begin{array}{l}
\left|S_{y} ;+\right\rangle \\
\left|S_{y} ;-\right\rangle
\end{array}\right)=\frac{1}{\sqrt{2}}\left(\begin{array}{cc}
1 & i \\
1 & -i
\end{array}\right)\left(\begin{array}{l}
\left|S_{z} ;+\right\rangle \\
\left|S_{z} ;-\right\rangle
\end{array}\right) .
$$

Note, that by symmetry the VUR inequality can also equally be written as

$$
q(1-q) \geq \frac{1}{4} \sin ^{2}\left(\tilde{\varphi}_{+}-\tilde{\varphi}_{-}\right),
$$

with

$$
e^{i \tilde{\varphi}_{ \pm}} \equiv \frac{\left\langle\phi \mid S_{x} ; \pm\right\rangle}{\left|\left\langle\phi \mid S_{x} ; \pm\right\rangle\right|}
$$


From (84) and (87) we see that the VUR does not pose any strong restriction between $\mathcal{P}$ and $\mathcal{Q}$. Since the phase factors $\varphi_{ \pm}\left(\right.$or $\tilde{\varphi}_{ \pm}$) do not enter the definition of $\mathcal{Q}$ (or $\mathcal{P}$ ), then for a fixed (but otherwise arbitrary) $q$ the VUR (84) can be in principle fulfilled by any $p \in[0.5,1]$. Of course, if the relative phase is known the restriction between $\mathcal{P}$ and $\mathcal{Q}$ is less trivial. On the other hand, the ITURs discussed above are far more specific in their constrains on values of $\mathcal{P}$ and $\mathcal{Q}$, see Tab. I. From the table we see that for given $\mathcal{P}$,

\begin{tabular}{|c|c|c|c|}
\hline$p$ & VUR $q \in$ & S-ITUR $q \in$ & R-ITUR $q \in$ \\
\hline \hline 0.5 & {$[0.067,0.933]$} & {$[0,1]$} & {$[0,1]$} \\
0.6 & {$[0.067,0.933]$} & {$[0.003,0.997]$} & {$[0.010,0.990]$} \\
0.7 & {$[0.067,0.933]$} & {$[0.017,0.983]$} & {$[0.042,0.958]$} \\
0.8 & {$[0.067,0.933]$} & {$[0.049,0.951]$} & {$[0.1,0.9]$} \\
0.9 & {$[0.067,0.933]$} & {$[0.121,0.879]$} & {$[0.2,0.8]$} \\
\hline
\end{tabular}

TABLE I: Comparison of three uncertainty relations: variance-based uncertainty relation (VUR) with $\tilde{\varphi}_{+}-\tilde{\varphi}_{-}=\pi / 6$, Shannon's information uncertainty relation (S-ITUR) and Rényi's information uncertainty relation (R-ITUR) for different values of $p$. In the respective columns one can see the peakedness of the distribution $\mathcal{Q}=(q,(1-q))$.

Rényi's ITUR improves on Shannon's ITUR. This is because the Rényi ITUR considered is more restrictive than Shannon's case. For instance, the marginal case $\mathcal{P}=(0.8,0.2)$ and $\mathcal{Q}=(0.951,0.049)$ that is allowed by Shannon's ITUR explicitly violates Rényi's ITUR and hence it cannot be realized (ITURs represent necessary conditions). Both Shannon's ITUR and Rényi's ITUR improve on VUR - unless some extra information about the relative wave-functions phase is provided. In Tab. I we find that when the relative phase is known, e.g., $\tilde{\varphi}_{+}-\tilde{\varphi}_{-}=\pi / 6$, Rényi's ITUR still improves on VUR for values $p=0.9$ and $p=0.8$ while Shannon's ITUR improves over VUR only for $p=0.9$.

\section{B. Continuous probabilities}

In view of the (continuous) ITUR from Section $\nabla$ the most prominent example of the Fourier transform is that between configuration and momentum space wave functions (analogously one can treat also other Fourier transform duals, such as the angular momentum and angle). In particular between $\psi(\mathbf{x})$ and $\hat{\psi}(\mathbf{p})$ hold two reciprocal relations

$$
\begin{aligned}
& \psi(\mathbf{x})=\int_{\mathbb{R}^{D}} e^{i \mathbf{p} \cdot \mathbf{x} / \hbar} \hat{\psi}(\mathbf{p}) \frac{d \mathbf{p}}{(2 \pi \hbar)^{D / 2}}, \\
& \hat{\psi}(\mathbf{p})=\int_{\mathbb{R}^{D}} e^{-i \mathbf{p} \cdot \mathbf{x} / \hbar} \psi(\mathbf{x}) \frac{d \mathbf{x}}{(2 \pi \hbar)^{D / 2}} .
\end{aligned}
$$


The Plancherel (or Riesz-Fischer) equality [61, 73] then implies that $\|\psi\|_{2}=\|\hat{\psi}\|_{2}=1$. Let us define new functions in (89), namely

$$
\begin{aligned}
& f^{(2)}(\mathbf{x})=(2 \pi \hbar)^{D / 4} \psi(\sqrt{2 \pi \hbar} \mathbf{x}) \\
& f^{(1)}(\mathbf{p})=(2 \pi \hbar)^{D / 4} \hat{\psi}(\sqrt{2 \pi \hbar} \mathbf{p})
\end{aligned}
$$

The factor $(2 \pi \hbar)^{D / 4}$ ensures that also the new functions are normalized (in sense of $\|\ldots\|_{2}$ ) to unity. With these we will have the same structure of the Fourier transform as in the Beckner-Babenko theorem in Section V. Consequently we can write the associated ITURs (53)-(54) in the form

$$
\begin{aligned}
\mathcal{I}_{1+t}\left(|\psi|^{2}\right)+\mathcal{I}_{1+r}\left(|\hat{\psi}|^{2}\right) & \geq D \log _{2}(\pi \hbar)+\frac{1}{r} \log _{2}(1+r)^{D / 2}+\frac{1}{t} \log _{2}(1+t)^{D / 2} \\
& =\frac{1}{r} \log _{2}\left(\frac{1+r}{\pi \hbar}\right)^{D / 2}+\frac{1}{t} \log _{2}\left(\frac{1+t}{\pi \hbar}\right)^{D / 2}
\end{aligned}
$$

or in the weaker form with the universal RHS

$$
\mathcal{I}_{1+t}\left(|\psi|^{2}\right)+\mathcal{I}_{1+r}\left(|\hat{\psi}|^{2}\right) \geq \log _{2}(2 \pi \hbar)^{D}
$$

In particular for Shannon's entropy the Hirschman inequality (57) acquires the form

$$
\mathcal{H}\left(|\psi|^{2}\right)+\mathcal{H}\left(|\hat{\psi}|^{2}\right) \geq \log _{2}(e \pi \hbar)^{D}
$$

In both (91) and (92) use was made of the mathematical identities

$$
\begin{aligned}
& \mathcal{I}_{\alpha}\left(\left|f^{(1)}\right|^{2}\right)=\mathcal{I}_{\alpha}\left(|\hat{\psi}|^{2}\right)-\frac{D}{2} \log _{2}(2 \pi \hbar) \\
& \mathcal{I}_{\alpha}\left(\left|f^{(2)}\right|^{2}\right)=\mathcal{I}_{\alpha}\left(|\psi|^{2}\right)-\frac{D}{2} \log _{2}(2 \pi \hbar)
\end{aligned}
$$

These two identities just state that the scaled PDFs $\left|f^{(1)}\right|^{2}$ and $\left|f^{(2)}\right|^{2}$ obtained from (90) are less peaked (and hence less informative) than the original PDFs $|\hat{\psi}|^{2}$ and $|\psi|^{2}$, respectively. Consequently, we increase our ignorance when passing from $\hat{\psi}$ to $f^{(1)}$, and from $\psi$ to $f^{(2)}$.

The inequality (92) (and similarly (91)) should be understood in the sense that by no quantum mechanical measurements it is possible to reduce the joint entropy in two canonically conjugate distributions $\mathcal{F}^{(1)}(\mathbf{p})=|\hat{\psi}(\mathbf{p})|$ and $\mathcal{F}^{(2)}(\mathbf{x})=|\psi(\mathbf{x})|$ below the level of $\log _{2}(2 \pi \hbar)^{D}$ bits.

Let us observe that in terms of the Rényi entropy power one can cast (91) into an equivalent form (cf. Eq. (59))

$$
N_{1+t}\left(|\psi|^{2}\right) N_{1+r}\left(|\hat{\psi}|^{2}\right) \geq \frac{\hbar^{2}}{4} .
$$




\section{Heavy tailed distributions}

If we wish to improve over the Shannon-Hirschman ITUR (57) we should find such a pair $\{r, t\}$ which provides a stronger restriction on the involved distributions than Shannon's case. In Section $\nabla$ we have already seen that this can indeed happen, e.g., for heavy tailed distributions. This fact will be now illustrated with Paretian or Lévy (stable) distributions. Such distributions represent, in general, a four parametric class of distributions that replace the rôle of the normal distribution in the central limit theorem in cases where the underlying single event distributions do not have one of the first two momenta. For computational simplicity (results can be obtained in a closed form) we will consider one of the Lévy stable distributions, namely the Cauchy-Lorentz distribution [11] which can be obtained from the wave function

$$
\hat{\psi}(x)=\sqrt{\frac{c}{\pi}} \sqrt{\frac{1}{c^{2}+(x-m)^{2}}} .
$$

The corresponding Fourier transform and respective PDFs are

$$
\begin{aligned}
& \hat{\psi}(p)=e^{-i m p / \hbar} \sqrt{\frac{2 c}{\pi^{2} \hbar}} K_{0}(c|p| / \hbar), \\
& \mathcal{F}^{(2)}(x)=\frac{c}{\pi} \frac{1}{c^{2}+(x-m)^{2}}, \\
& \mathcal{F}^{(1)}(p)=\frac{2 c}{\pi^{2} \hbar} K_{0}^{2}(c|p| / \hbar),
\end{aligned}
$$

and the ensuing Shannon and Rényi entropies are

$$
\begin{aligned}
& \mathcal{H}\left(\mathcal{F}^{(1)}\right)=\log _{2}\left(\pi^{2} \hbar / 2 c\right)-\frac{8}{\pi^{2}} 2.8945, \quad \mathcal{H}\left(\mathcal{F}^{(2)}\right)=\log _{2}(4 c \pi), \\
& \mathcal{I}_{1 / 2}\left(\mathcal{F}^{(1)}\right)=\log _{2}(2 \hbar / c), \quad \mathcal{I}_{\infty}\left(\mathcal{F}^{(2)}\right)=\log _{2}(c \pi) .
\end{aligned}
$$

With these results we can immediately write the associated ITURs, namely

$$
\begin{aligned}
& \mathcal{H}\left(\mathcal{F}^{(1)}\right)+\mathcal{H}\left(\mathcal{F}^{(2)}\right)=\log _{2}\left(2 \pi^{3} \hbar\right)-\frac{8}{\pi^{2}} 2.8945>\log _{2}(e \pi \hbar), \\
& \mathcal{I}_{1 / 2}\left(\mathcal{F}^{(1)}\right)+\mathcal{I}_{\infty}\left(\mathcal{F}^{(2)}\right)=\log _{2}(2 \pi \hbar) .
\end{aligned}
$$

So what can be concluded from these relations? First we notice that the ITUR (102) saturates the inequality (91) while the Shannon ITUR (101) does not saturate the corresponding Hirschman inequality (93). In fact, if we rewrite (101)-(102) in the language of Rényi entropy powers, we obtain

$$
\begin{aligned}
& N\left(\mathcal{F}^{(1)}\right) N\left(\mathcal{F}^{(2)}\right)>\frac{\hbar^{2}}{4} \\
& N_{1 / 2}\left(\mathcal{F}^{(1)}\right) N_{\infty}\left(\mathcal{F}^{(2)}\right)=\frac{\hbar^{2}}{4} .
\end{aligned}
$$


Since the Rényi ITUR puts a definite constraint between $\mathcal{F}^{(2)}$ and $\mathcal{F}^{(1)}$ it clearly improves over the Shannon ITUR (which is less specific). In addition, while (103) indicates that one could still find another $\mathcal{F}^{(2)}$ for a given fixed $\mathcal{F}^{(1)}$ that would lower the LHS of the Shannon entropy power inequality, the relation (104) forbids such a situation to happen without increasing uncertainty in the Rényi ITUR. By increasing the uncertainty, however, the definite constraint between $\mathcal{F}^{(2)}$ and $\mathcal{F}^{(1)}$ will get lost.

It should be stressed, that in general the Rényi ITUR is not symmetric. However, in the case at hand the situation is quite interesting. One can easily check that $\mathcal{I}_{1 / 2}\left(\mathcal{F}^{(2)}\right)=\infty$ and $\mathcal{I}_{\infty}\left(\mathcal{F}^{(1)}\right)=-\infty$, and so the Rényi ITUR is indeterminate. This result deserves two comments. First, the extremal values of $\mathcal{I}_{1 / 2}\left(\mathcal{F}^{(2)}\right)$ and $\mathcal{I}_{\infty}\left(\mathcal{F}^{(1)}\right)$ can be easily understood. From the very formulation of the RE one can see that for $\alpha>1$ the non-linearly nature of the RE tends to emphasize the more probable parts of the PDF (typically the middle parts) while for $\alpha<1$ ) the less probable parts of the PDF (typically the tails) are accentuated. In other words, $\mathcal{I}_{1 / 2}$ mainly carries information on the rare events while $\mathcal{I}_{\infty}$ on the common events. In particular, if one starts from a strongly leptocurtic distribution (such as $\mathcal{F}^{(1)}$ ) then $\mathcal{I}_{\infty}$ effectively works with the PDF that is sharply (almost $\delta$-function) peaked. In this respect ignorance about the peak is minimal, which in turn corresponds to the minimal $\mathrm{RE}$ which for continuous distributions is $-\infty$. For heavy tailed distributions (such as $\mathcal{F}^{(2)}$ ) the $\mathrm{RE} \mathcal{I}_{1 / 2}$ works effectively with a very flat (almost equiprobable) PDF which yields maximal ignorance about the tail. For continuous distributions the related information of the order $\alpha=1 / 2$ is thus $\infty$.

Second, one can make sense of the indeterminate form of the Rényi ITUR by putting a regulator on the real $x$ axis. In particular we can assume that $\int_{-\infty}^{\infty} d x \ldots \mapsto \int_{-R}^{R} d x \ldots$ With this we obtain to leading order in $R$

$$
\begin{aligned}
& \mathcal{I}_{1 / 2}\left(\mathcal{F}^{(2)}\right)=2 \log _{2}\left(\sqrt{\frac{c}{\pi}} \log \left(4 R^{2} / c^{2}\right)\right) \\
& \mathcal{I}_{\infty}\left(\mathcal{F}^{(1)}\right)=-\log _{2}\left(\frac{2 c}{\hbar \pi^{2}} K_{0}^{2}(c / R)\right) .
\end{aligned}
$$

In the associated ITUR the unwanted divergent terms cancel and we end up with the final result

$$
\mathcal{I}_{1 / 2}\left(\mathcal{F}^{(2)}\right)+\mathcal{I}_{\infty}\left(\mathcal{F}^{(1)}\right) \stackrel{R \rightarrow \infty}{=} \log _{2}(2 \pi \hbar)
$$

which again, rather surprisingly, saturates the information bound.

It is also interesting to observe that while the variance in momentum $\left\langle(\triangle p)^{2}\right\rangle_{\psi}=$ $\hbar^{2} \pi / 16 c^{2}$, the variance in position $\left\langle(\triangle x)^{2}\right\rangle_{\psi}=\infty$ (which is symptomatic of Lévy stable distributions) and hence the Schrödinger-Robertson VUR is completely uninformative. Similar conclusions can be also reached with the Lévy-Smirnov distribution which is used in fractional QM [82, 83] and which can be obtained from the wave function

$$
\psi(x)=\left(\frac{c}{2 \pi}\right)^{1 / 4} \exp \left(-\frac{c}{4}(x-m)^{-1}+\frac{i}{\hbar} p_{0} x\right) /(x-m)^{3 / 4} .
$$


Let us finally note that the meaning of the ITUR (91) (and (92)) is rather different from the momentum-position VUR. The difference is due to the fact that the two measures of uncertainty (namely variance and Rényi's entropy) are left unaltered by very different types of PDF modifications. While both the variance of a probability distribution and Rényi entropy are translation invariant (i.e., invariant under the shift of the mean value of the distribution by a constant), Rényi entropy is, in addition, invariant under the piecewise reshaping of the wave function. Particularly PDF's $\varrho^{(2)}(x)=\left|\psi^{(1)}(x)\right|^{2}$ and $\varrho^{(2)}(x)=\left|\bar{\psi}^{(2)}(x)\right|^{2}$ with the wave function

$$
\bar{\psi}^{(2)}(x)=\sum_{n \in \mathbb{N}} \chi_{[n d x,(n+1) d x]} \psi^{(2)}\left(x_{\sigma(n)}\right),
$$

$\left(\chi_{[a, b]}\right.$ is the indicator function of the interval $[a, b]$ in $\mathbb{R}$ and $\sigma(n)$ is an arbitrary permutation of the set of all $n \in \mathbb{N}$ ) yield the same Rényi entropy. In other words, Rényi entropy is invariant under cutting up the original PDF $\varrho^{(2)}(x)$ into infinitesimal pieces under the original curve and reshuffling or separating them in an arbitrary manner. Also the Rényi entropy for corresponding Fourier transformed wave functions are unchanged when passing from $\varrho^{(1)}(\mathbf{p})$ to $\bar{\varrho}^{(1)}(\mathbf{p})$. This indicates that the corresponding ITUR will not change under such a reshuffling. This fact will be illustrated in the following subsection.

\section{Schrödinger cat states}

Another relevant situation when the continuous ITUR improves on the VUR occurs for coherent state superpositions (CSS), also called Schrödinger cat states. These states have the form

$$
\left|C S S_{ \pm}(\beta)\right\rangle=N_{\beta}^{ \pm}(|\beta\rangle \pm|-\beta\rangle)
$$

where $|\beta\rangle$ is the ordinary Glauber coherent state with the amplitude $\beta$ and

$$
N_{\beta}^{ \pm}=1 / \sqrt{2\left(1 \pm \mathrm{e}^{-2 \beta^{2}}\right)}
$$

is the normalization factor. Such states have been created in the laboratory [74] and are of interest in studies of the quantum to classical transition as well as quantum metrology [75, 76]. For definiteness we shall consider only the $\left|C S S_{+}(\beta)\right\rangle$ state, though the qualitative statements will equally hold also for $\left|C S S_{-}(\beta)\right\rangle$. The operator corresponding to different phase quadratures of this state is

$$
\hat{X}_{\theta}=\left(\hat{b} e^{-i \theta}+\hat{b}^{\dagger} e^{i \theta}\right) / 2
$$

where $\hat{b}^{\dagger}$ and $\hat{b}$ are respectively the creation and annihilation operators for a photon in the coherent state mode. Note that the eigenvalues of these operators are unitless and do not depend on $\hbar$ as was the case with the other examples. We shall be concerned with the orthogonal quadratures $\hat{X}_{0}$ and $\hat{X}_{\pi / 2}$, which form a pair of conjugate observables with the 
commutation relation $\left[\hat{X}_{0}, \hat{X}_{\pi / 2}\right]=i / 2$. If we take $\left|x_{0}\right\rangle$ and $\left|x_{\pi / 2}\right\rangle$ to be eigenstates of $\hat{X}_{0}$ and $\hat{X}_{\pi / 2}$ we can represent (109) in these bases as

$$
\begin{aligned}
\left\langle x_{0} \mid C S S_{+}(\beta)\right\rangle & =N_{\beta}^{+}\left(\left\langle x_{0} \mid \beta\right\rangle+\left\langle x_{0} \mid-\beta\right\rangle\right) \\
& =\frac{2 N_{\beta}^{+}}{\pi^{\frac{1}{4}}} \cosh \left(\sqrt{2} \beta x_{0}\right) \exp \left[-\frac{1}{2} x_{0}^{2}-\beta^{2}\right], \\
\left\langle x_{\pi / 2} \mid C S S_{+}(\beta)\right\rangle & =N_{\beta}^{+}\left(\left\langle x_{\pi / 2} \mid \beta\right\rangle+\left\langle x_{\pi / 2} \mid-\beta\right\rangle\right) \\
& =\frac{2 N_{\beta}^{+}}{\pi^{\frac{1}{4}}} \cos \left(\sqrt{2} \beta x_{\pi / 2}\right) \exp \left[-\frac{1}{2} x_{\pi / 2}^{2}\right] .
\end{aligned}
$$

The corresponding probability distributions

$$
\begin{aligned}
\mathcal{F}^{(2)}\left(x_{0}\right) & =\left\langle x_{0} \mid C S S_{+}(\beta)\right\rangle\left\langle C S S_{+}(\beta) \mid x_{0}\right\rangle \\
\mathcal{F}^{(1)}\left(x_{\pi / 2}\right) & =\left\langle x_{\pi / 2} \mid C S S_{+}(\beta)\right\rangle\left\langle C S S_{+}(\beta) \mid x_{\pi / 2}\right\rangle
\end{aligned}
$$

can be experimentally accessed with homodyne detections.

The ensuing values of Shannon and Rényi entropies are depicted in Fig. $7 \mathrm{~h}$ as functions of $\beta$. Curve (i) is the Shannon ITUR, $\mathcal{H}\left(\mathcal{F}^{(2)}\right)+\mathcal{H}\left(\mathcal{F}^{(1)}\right)$, and the dashed curve (ii) depicts the bound for the Shannon ITUR, i.e. $\log _{2}(e \pi)$. We see that the bound is saturated for small $\beta$ (as should be expected for a single Gaussian wave packet) and gets worse as $\beta$ is increased (and information about the localization worsens), but eventually saturates at some value above the bound (when two Gaussian wave packets no longer overlap). The plateau is a consequence of the mentioned fact that the $\mathrm{RE}$ is immune to piecewise rearrangements of the distributions. Namely, a PDF consisting of two well separated wave packets has the same RE irrespective of the mutual distance. This holds true also for the associated $\mathcal{F}^{(1)}$ PDF.

The other curves are for the Rényi ITURs: (iii) is $\mathcal{I}_{1 / 2}\left(\mathcal{F}^{(1)}\right)+\mathcal{I}_{\infty}\left(\mathcal{F}^{(2)}\right)$ where the qualitative behavior is similar as in the Shannon case. In this situation we see that the plateau forms earlier, which indicates that information about the peak part (i.e. $\mathcal{I}_{\infty}$ ) starts to saturate earlier than in the Shannon entropy case, which democratically takes into account all parts of the underlying PDF. The dashed curve (iv) is the other way round, i.e. $\mathcal{I}_{1 / 2}\left(\mathcal{F}^{(2)}\right)+\mathcal{I}_{\infty}\left(\mathcal{F}^{(1)}\right)$. The faint solid line overlapping with the dashed line (iv) is the Rényi entropy bound, $\log _{2}(2 \pi)$. We see that both configurations saturate the bound for small $\beta$, but the dashed one saturates the bound for all $\beta$. The saturation of the information bound can be attributed to the interplay between the degradation of information on the ail parts of $\mathcal{F}^{(2)}$ carried by $\mathcal{I}_{1 / 2}\left(\mathcal{F}^{(2)}\right)$ and the gain of information on the central part of $\mathcal{F}^{(2)}$ conveyed by $\mathcal{I}_{\infty}\left(\mathcal{F}^{(1)}\right)$. Interestingly enough, the rate of change (in $\beta$ ) for both REs is identical but opposite in sign thus yielding a $\beta$-independent ITUR.

For the same reasons as in the previous subsection the R-ITUR outperforms the S-ITUR. In addition, we again note that while the variance $\left(\Delta x_{\pi / 2}\right)^{2}$ is finite for arbitrary $\beta$,

$$
\left\langle C S S_{+}(\beta)\left|\left(\triangle x_{\pi / 2}\right)^{2}\right| C S S_{+}(\beta)\right\rangle=\left(N_{\beta}^{+}\right)^{2}\left[1+e^{-2 \beta^{2}}\left(1-4 \beta^{2}\right)\right],
$$



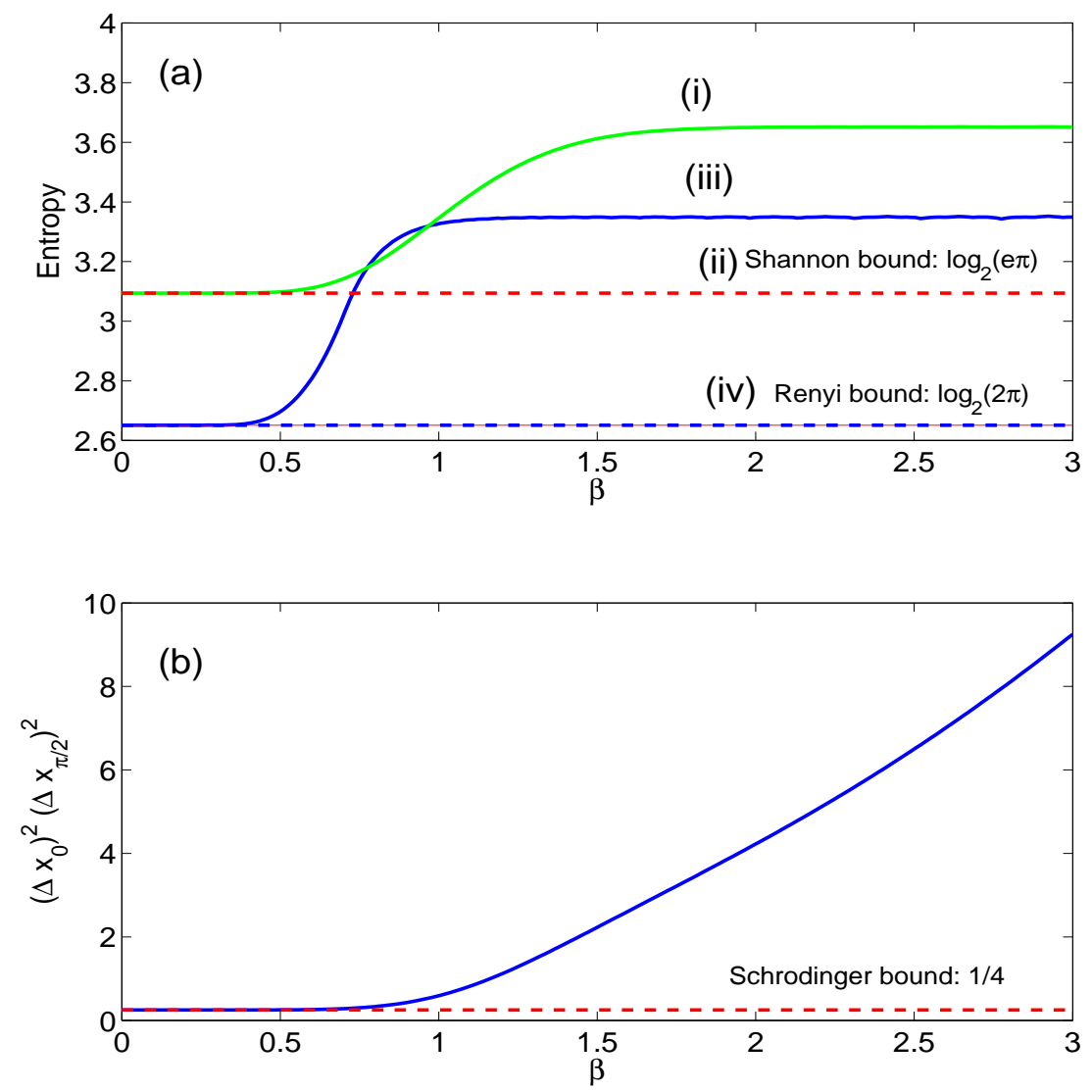

FIG. 7: a) Plot of different entropies for $\left|C S S_{+}(\beta)\right\rangle$ as a function of $\beta$. (i) Shannon ITUR, $\mathcal{H}\left(\mathcal{F}^{(2)}\right)+\mathcal{H}\left(\mathcal{F}^{(1)}\right)$, (ii) bound for the Shannon ITUR, $\log _{2}(e \pi)$ (iii) Renyi ITUR, $\mathcal{I}_{1 / 2}\left(\mathcal{F}^{(1)}\right)+$ $\mathcal{I}_{\infty}\left(\mathcal{F}^{(2)}\right)$, and (iv) the other way round, $\mathcal{I}_{1 / 2}\left(\mathcal{F}^{(2)}\right)+\mathcal{I}_{\infty}\left(\mathcal{F}^{(1)}\right)$. Also shown as a faint solid line overlapping with (iv) is the Rényi ITUR bound, $\log _{2}(2 \pi)$. b) Plot of the Robertson-Schrödinger VUR for $\left|C S S_{+}(\beta)\right\rangle$ as a function of $\beta$ (solid curve) and its bound (dashed curve).

the variance of the conjugate quadrature

$$
\left\langle C S S_{+}(\beta)\left|\left(\triangle x_{0}\right)^{2}\right| C S S_{+}(\beta)\right\rangle=\left(N_{\beta}^{+}\right)^{2}\left[1+e^{-2 \beta^{2}}+4 \beta^{2}\right],
$$

can be arbitrary large subject to the value of $\beta$ (see Fig. $7 \mathrm{~b}$ ). In this respect the SchrödingerRobertson VUR again tends to be uninformative for large values of $\beta$ (i.e., for a large wavepacket separation). Fig. 7 b shows the product of the quadrature variances (solid curve) along with the Robertson-Schrödinger bound (dashed curve). We see that the bound is saturated for small coherent state amplitudes $(\beta)$ but gets progressively worse as $\beta$ is increased. 


\section{CONCLUSIONS AND OUTLOOK}

In this paper we have generalized the information theoretic uncertainty relations that have been previously developed in Refs. [7 9, 12, 14] to include generalized information measures of Rényi and RE-based entropy powers. To put some flesh on the bones we have applied these generalized ITURs to a simple two-level quantum system (in the discrete-probability case) and to quantum-mechanical systems with heavy-tailed distributions and Schrödinger cat states (in the continuous-probability case). An improvement of the Rényi ITUR over both the Robertson-Schrödinger VUR and the Shannon ITUR was demonstrated in all the aforementioned cases.

In connection with the discrete-probability ITUR we have also highlighted a geometric interpretation by showing that the lower bound on information content (or uncertainty) inherent in the ITUR is higher, the smaller is the distance to singularity of the transformation matrix connecting eigenstates of the two involved observables.

The presented ITURs hold promise precisely because a large part of the structure of quantum theory has an information theoretic underpinning (see, e.g., Refs. [28, 77]). In this connection it should be stressed that, ITURs in general should play a central rôle, for instance, in quantum cryptography or in the theory of quantum computers, particularly in connection with quantum error-correcting codes, communication and algorithmic complexities. In fact, information measures such as Rényi's entropy are used not because of intuitively pleasing aspects of their definitions but because there exist various (classical and quantum) coding theorems [26, 39] which endow them with an operational (that is, experimentally verifiable) meaning. While coding theorems do exist for Shannon, Rényi or Holevo entropies, there are (as yet) no such theorems for Tsallis, Kaniadakis, Naudts and other currently popular entropies. The information theoretic significance of such entropies is thus not obvious, though in the literature one can find, for instance, a Tsallis entropy based version of the uncertainty relations [78].

Though our reasoning was done in the framework of the classical (non-quantum) information theory, it is perhaps fair to mention that there exist various generalizations of Rényi entropies to the quantum setting. Most prominent among theses are Petz's quasientropies [84] and Renner's conditional min-, max-, and collision entropy [85]. Nevertheless, the situation in the quantum context is much less satisfactory in that these generalizations do not have any operational underpinning and, in addition, they are incompatible with each other in number of ways. For instance, whereas the classical conditional min-entropy can be naturally derived from the Rényi divergence, this does not hold for their quantum counterparts. At present there is no obvious generalization of the Rényi entropy power in the quantum framework and hence it is not obvious in what sense one should interpret the prospective ITUR. All these aforementioned issues are currently under active investigation.

Let us finally make a few comments concerning the connection of the entropy power with Fisher information. Fisher information was originally employed by Stam [67] in his proof of the Shannon entropy power inequality. Interestingly enough, one can use either the entropy power inequality or the Cramér-Rao inequality and logarithmic Sobolev inequality to re-derive the usual Robertson-Schrödinger VUR. While the generalized ITUR presented 
here can be derived from the generalized entropy power inequality (as both are basically appropriate restatements of Young's theorem), the connection with Fisher information (or some of its generalizations) is not yet known. The corresponding extension of our approach in this direction would be worth pursuing particularly in view of the natural manner in which RE is used both in inference theory and ITUR formulation.

Last, but not least, the Riesz-Thorin and Beckner-Babebko inequalities that we have utilized in Sections [V and V belong to a set of inequalities commonly known as $\mathcal{L}^{p}$-interpolation theorems [65]. It would be interesting to see whether one can sharpen our analysis from Section [V] by using the Marcinkiewicz interpolation theorem [65], which in a sense represents the deepest interpolation theorem. In particular, the latter avoids entirely the Riesz convexity theorem which was key in our proof. Work along these lines is presently in progress.

\section{Acknowledgments}

P.J. would like to gratefully acknowledge stimulating discussions with H. Kleinert, P. Harremoës and D. Brody. This work was supported by GAČR Grant No. P402/12/J077.

\section{Appendix A}

In this Appendix we introduce the (generalized) Young inequality and derive some related inequalities. Since the actual proof of Young's inequality is rather involved we provide here only its statement. The reader can find the proof together with further details, e.g., in Ref. [72].

Theorem A.1 (Young's theorem) Let $q, p, r>0$ represent Hölder triple, i.e.,

$$
\frac{1}{q}+\frac{1}{p}=1+\frac{1}{r}
$$

and let $\mathcal{F} \in \ell^{q}\left(\mathbb{R}^{D}\right)$ and $\mathcal{G} \in \ell^{p}\left(\mathbb{R}^{D}\right)$ are two non-negative functions, then

$$
\|\mathcal{F} * \mathcal{G}\|_{r} \geq C^{D}\|\mathcal{F}\|_{q}\|\mathcal{G}\|_{p}
$$

for $q, p, r \geq 1$ and

$$
\|\mathcal{F} * \mathcal{G}\|_{r} \leq C^{D}\|\mathcal{F}\|_{q}\|\mathcal{G}\|_{p}
$$

for $q, p, r \leq 1$. The constant $C$ is

$$
C=C_{p} C_{q} / C_{r} \quad \text { with } \quad C_{x}^{2}=\frac{|x|^{1 / x}}{\left|x^{\prime}\right|^{1 / x^{\prime}}}
$$

Here $x$ and $x^{\prime}$ are Hölder conjugates. Symbol * denotes a convolution. 
Young inequality allows to prove very quickly the Hausdorff-Young inequalities which are instrumental in obtaining various Fourier-type uncertainty relations. In fact, the following chain of reasons holds

$$
\|\mathcal{F} * \delta\|_{r} \geq C^{D}\|\mathcal{F}\|_{q}\|\delta\|_{p}=C^{D}\|\mathcal{F}\|_{q} V_{R}^{(p-1) / p}
$$

Here we have used the fact that for the $\delta$ function

$$
\|\delta\|_{p}=\left[\int_{\mathbb{R}^{D}} d \mathbf{x} \delta^{p}(\mathbf{x})\right]^{1 / p}=\left[\int_{\mathbb{R}^{D}} d \mathbf{x} \delta(\mathbf{x}) \delta^{p-1}(0)\right]^{1 / p}=V_{R}^{(p-1) / p} .
$$

In the derivation we have utilized that

$$
\delta(0)=\int_{\mathbb{R}^{D}} d \mathbf{x} e^{i \mathbf{p} \cdot \mathbf{0}}=V_{R}
$$

Subindex $R$ indicates that the volume is regularized, i.e., we approximate the actual volume of $\mathbb{R}^{D}$ with a $D$-dimensional ball of the radius $R$, where $R$ is arbitrarily large but fixed. At the end of calculations we send $R$ to infinity. We should also stress that in (A33) an implicit assumption was made that $q, p, r \geq 1$.

The norm $\|\mathcal{F} * \delta\|_{r}$ fulfills yet another inequality, namely

$$
\|\mathcal{F} * \delta\|_{r}=\left[\int_{\mathbb{R}^{D}} d \mathbf{x}\left(\int_{\mathbb{R}^{D}} d \mathbf{p} e^{-i \mathbf{p} \cdot \mathbf{x}} \hat{\mathcal{F}}(\mathbf{p})\right)^{r}\right]^{1 / r} \leq\|\hat{\mathcal{F}}\|_{n} V_{R}^{1 / n^{\prime}+1 / r}
$$

where we have used the Hölder inequality

$$
\int_{\mathbb{R}^{D}} d \mathbf{p} e^{-i \mathbf{p} \cdot \mathbf{x}} \hat{\mathcal{F}}(\mathbf{p})=\left|\int_{\mathbb{R}^{D}} d \mathbf{p} e^{-i \mathbf{p} \cdot \mathbf{x}} \hat{\mathcal{F}}(\mathbf{p})\right| \leq\|\hat{\mathcal{F}}\|_{n}\left\|e^{-i \mathbf{p} \cdot \mathbf{x}}\right\|_{n^{\prime}}=\|\hat{\mathcal{F}}\|_{n} V_{R}^{1 / n^{\prime}}
$$

with $n$ and $n^{\prime}$ being Hölder's conjugates $(n \geq 1)$.

Comparing (A33) with (A4) gives the inequality

$$
\|\hat{\mathcal{F}}\|_{n} V_{R}^{1 / n^{\prime}+1 / r} \geq C^{D}\|\mathcal{F}\|_{q} V_{R}^{(p-1) / p} .
$$

The volumes will mutually cancel provided $1 / n^{\prime}+1 / r+1 / p=1$, or equivalently, when $1 / n^{\prime}=1 / q-2 / r$. With this we can rewrite (A5) as

$$
\|\hat{\mathcal{F}}\|_{n} \geq C^{D}\|\mathcal{F}\|_{q} \geq C^{D}\|\mathcal{F}\|_{n^{\prime}}
$$

The last inequality results from Hölder's inequality:

$$
\|\mathcal{F}\|_{a} \geq\|\mathcal{F}\|_{b} \quad \text { when } \quad a \leq b .
$$

In fact, in the limit $r \rightarrow \infty$ the last inequality in $(\underline{\mathrm{A} 6})$ is saturated and $C \stackrel{r \rightarrow \infty}{\rightarrow} 1$. Consequently we get the Hausdorff-Young inequality in the form

$$
\|\hat{\mathcal{F}}\|_{n} \geq\|\mathcal{F}\|_{n^{\prime}}
$$




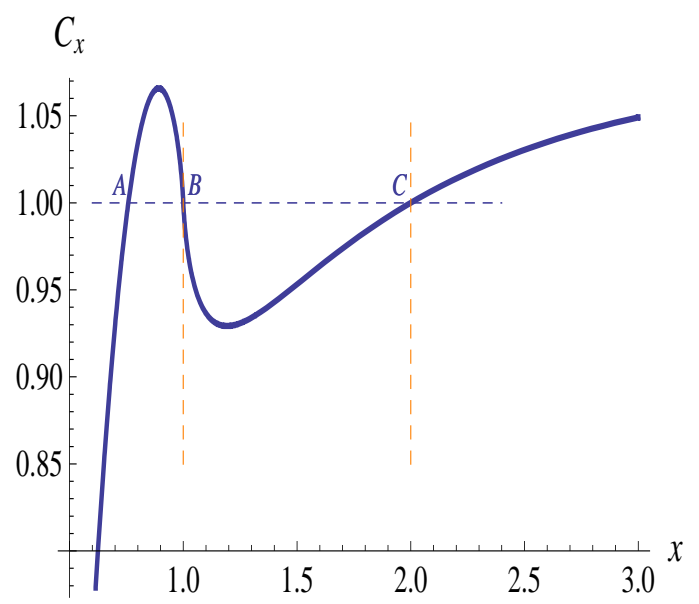

FIG. 8: Dependence of the constant $C_{x}$ on the Hölder parameter $x$. When $x$ is between points $B$ and $C$, i.e., when $x \in[1,2]$ than $C_{x} \leq 1$. For $x \leq A$ is $C_{x}$ also smaller than 1 but such $x$ are excluded by the fact that $x$ must be $\geq 1$.

This inequality holds, of course, only when $q \geq n^{\prime}$ (cf. equation (A7)), i.e., when $n \geq$ $q /(q-1)$. Since $q \geq 1$ we have that $n \in[1,2]$. Should we have started in our derivation with $\hat{\mathcal{F}}$ instead of $\mathcal{F}$ we would have obtain the reverse inequality

$$
\|\mathcal{F}\|_{n} \geq\|\hat{\mathcal{F}}\|_{n^{\prime}} .
$$

Inequalities, (A8) and (A9) are known as classical Hausdorff-Young inequalities [61]. Note that in the spacial case when $n=2$ we have also $n^{\prime}=2$ and equations (A8) - (A9) together imply equality:

$$
\|\mathcal{F}\|_{2}=\|\hat{\mathcal{F}}\|_{2} .
$$

This is known as the Plancherel (or Riesz-Fischer) equality [61, 73].

It should be noted that the Beckner-Babenko inequality from Section 5 improves upon the Hausdorff-Young inequalities. This is because $C_{x} \leq 1$ for $x \in[1,2]$, see Fig. \& The Beckner-Babenko inequality follows easily from Young's inequality. Indeed, assume that there exists a (possibly $p$-dependent) constant $k(p) \leq 1$, such that

$$
k(p)\|\mathcal{F}\|_{p} \geq\|\hat{\mathcal{F}}\|_{p^{\prime}} \quad \text { and } \quad k(p)\|\hat{\mathcal{F}}\|_{p} \geq\|\mathcal{F}\|_{p^{\prime}} .
$$

The constant $k(p)$ can be easily found by writing

$$
k(r)\|\mathcal{F} * \mathcal{G}\|_{r} \geq\|\hat{\mathcal{F}} \hat{\mathcal{G}}\|_{r^{\prime}} \geq\|\hat{\mathcal{F}}\|_{q^{\prime}}\|\hat{\mathcal{G}}\|_{p^{\prime}} \geq\left[k\left(q^{\prime}\right)\right]^{-1}\|\mathcal{F}\|_{q}\left[k\left(p^{\prime}\right)\right]^{-1}\|\mathcal{G}\|_{p},
$$

which gives

$$
\|\mathcal{F} * \mathcal{G}\|_{r} \geq[k(r)]^{-1}\left[k\left(q^{\prime}\right)\right]^{-1}\left[k\left(p^{\prime}\right)\right]^{-1}\|\mathcal{F}\|_{q}\|\mathcal{G}\|_{p} .
$$

The middle inequality in (A12) is the Hölder inequality that is valid for $1 / r^{\prime}=1 / p^{\prime}+1 / q^{\prime}$ (i.e., for $1 / p+1 / q=1 / r+1$ ). Comparison of (A13) with (A1) gives the equation

$$
[k(r)]^{-1}\left[k\left(q^{\prime}\right)\right]^{-1}\left[k\left(p^{\prime}\right)\right]^{-1}=C^{D}=\left[C_{q} C_{p} / C_{r}\right]^{D}=\left[1 / C_{q^{\prime}} C_{p^{\prime}} C_{r}\right]^{D} .
$$


This is clearly solved with $k(p)=C_{p}^{D}$. By choosing $p \in[1,2]$ we get improvement over the Hausdorff-Young inequalities.

[1] W. Heisenberg, Z. Phys. 43 (1927) 172.

[2] W. Heisenberg, The Physical Principles of the Quantum Theory (Dover, New York, 1949).

[3] J. Erhart, S. Sponar, G. Sulyok, G. Badurek, M. Ozawa and Y. Hasegawa, Nature Phys. 8 (2012) 185.

[4] E.H. Kennard, Zeitschrift für Physik 44 (1927) 326.

[5] H.P. Robertson, Phys. Rev. 34 (1929) 163.

[6] E. Schrödinger, Sitzungsber. Preuss. Acad. Wiss. 24 (1930) 296.

[7] I. Białynicky-Birula and J. Mycielski, Commun. Math. Phys. 44 (1975) 129.

[8] D.L. Deutsch, Phys. Rev. Lett. 50 (1983) 631.

[9] H. Maassen and J.B.M. Uffink, Phys. Rev. Lett. 60 (1988) 1103.

[10] P. Lévy, Théorie de l'Addition des Variables Aléatoires (Gauthier-Villars, Paris, 1937 \& 1954).

[11] W. Feller, An Introduction to Probability Theory and Its Applications, Vol. II (John Wiley, London, 1966).

[12] J.B.M. Uffink, Measures of Uncertainty and the Uncertainty Principle, PhD thesis, University of Utrecht, Januari 1990; http://www.phys.uu.nl/igg/jos/publications/proefschrift.pdf

[13] H.E. Montgomery, Jr., Chemical Educator 7 (2002) 334.

[14] I.I. Hirschman, Jr., Amer. J. Math. 79 (1957) 152.

[15] This distribution is in physics also known as the Breit-Wigner distribution. It is particularly relevant in nuclear and particle physics, and in spectroscopy.

[16] L. Lyons, Statistics for Nuclear and Particle Physicists (Cambridge University Press, Cambridge, 1989).

[17] P.A.M. Dirac, The principles of Quantum Mechanics (Claredon Pres, Oxford, 1958).

[18] D.L. Donoho and P.B. Stark, SIAM J. Appl. Math. 49 (1989) 906.

[19] D. Mustard, Austral. Math. Soc. Ser. B 33 (1991) 180.

[20] B. Lesche, J. Stat. Phys. 27 (1982) 419.

[21] P. Jizba and T. Arimitsu, Ann. Phys. 312 (2004) 17.

[22] A. Rényi, Probability Theory (North-Holland, Amsterdam, 1970); Selected Papers of Alfred Rényi, Vol.2 (Akad émia Kiado, Budapest, 1976).

[23] C.E. Shannon A mathematical theory of communication, Bell Syst. Tech. J. 27 (1948) 379; 623.

[24] M.H. Costa, IEEE Trans. Inform. Theory, 31 (1985) 751.

[25] I. Csiszár, IEEE Trans. Inform. Theory 41 (1995) 26.

[26] L.L. Campbell, Informat. Contr. 8 (1965) 423.

[27] M.B. Baer, Rényi to Rényi - source coding under siege, in: Proc., 2006 IEEE Int. Symp. on Information Theory, July 14, 2006, pp. 1258-1262.

[28] M. Nielsen and I.L. Chuang, Quantum Computation and Quantum Information (Cambridge Un. Press, Cambridge, 2000). 
[29] see e.g., V.V. Dodonov, E.V. Kurmyshev and V.I. Man'ko, Phys. Lett. A 79 (1980) 150.

[30] J.M. Lévy-Leblond, Ann. Phys. 101 (1976) 319.

[31] P. Carruthers and M.M. Nietto, Rev. Mod. Phys. 40 (1968) 411.

[32] L. Mandelstam and I.G. Tamm, J. Phys. USSR 9 (1945) 249.

[33] W.H. Luisell, Quantum Statistical Theory of Radiation (Wiley, New York, 1973).

[34] J.L. Synge, Proc. R. Society London A 325 (1971) 151.

[35] M. Ozawa, Phys. Rev. A 67 (2003) 042105.

[36] M. Ozawa, J. Opt. B 7 (2005) 672.

[37] C. Cachin, Entropy Measures and Unconditional Security in Cryptography, PhD thesis, ETH Zurich, May 1997; ftp://ftp.inf.ethz.ch/pub/publications/dissertations/th12187.ps.gz.

[38] C.H Bennett, G. Brassard, C. Crepeau, and U.M. Maurer, IEEE Trans. Inform. Theory 41 (1995) 1915.

[39] P. Jizba, H. Kleinert and M. Shefaat, Physica A 391 (2012) 2971.

[40] P. Jizba and J. Korbel, arXiv:1401.3316 [q-fin.ST].

[41] M. Hayashi, IEEE Trans. Inform. Theory 57 (2011) 3989.

[42] G. Adesso, D. Girolami and A. Serafini, Phys. Rev. Lett. 109 (2012) 190502.

[43] S. Kullback and R. Leibler, Ann. Math. Statist. 22 (1951) 79.

[44] T. Arimitsu and N. Arimitsu, J. Phys. A: Math. Gen. 33 (2000) L235.

[45] T. Arimitsu and N. Arimitsu, Physica A 295 (2001) (2001) 673.

[46] T. Arimitsu and N. Arimitsu, Physica A 305 (2002) 218.

[47] T. Arimitsu and N. Arimitsu, Condens. Matter 14 (2002) 2237.

[48] T.C. Halsey, M.H. Jensen, L.P. Kadanoff, I. Procaccia and B.I. Shraiman, Phys. Rev. A 33 (1986) 1141.

[49] M.H. Jensen, L.P. Kadanoff, A. Libchaber, I. Procaccia and J. Stavans, Phys. Rev. Lett. 55 (1985) 2798.

[50] K. Tomita, H. Hata, T. Horita, H. Mori and T. Morita, Prog. Theor. Phys. 80 (1988) 963.

[51] H.G.E. Hentschel and I. Procaccia, Physica D 8 (1983) 435.

[52] M. Riesz, Acta. Math. 49 (1926) 465.

[53] O. Thorin, Kungl. Fysiogr. Sällsk. i Lund Förh. 8 (1939) 14 (= Medd. Lunds Univ. Mat. Sem. 4 (1939) 1).

[54] T. Cover and J. A. Thomas. Elements of Information Theory, (Wiley, London, 1991).

[55] S. Verdu and D. Guo. IEEE Trans. Inform. Theory 52(2006) 2165.

[56] A. Dembo, IEEE Trans. Inform. Theory 35 (1989) 887.

[57] N.M. Blachman, IEEE Trans. Inform. Theory 11 (1965) 267.

[58] R.J. Gardner, Bull. Amer. Math. Soc. 39 (2002) 355.

[59] S.M. Kay Fundamentals of Statistical Signal Processing, Volume I: Estimation Theory, ( Prentice Hall, New York, 1993).

[60] Note that, since $a_{i j} \in S O(n)$, then $a_{i j}^{-1}=a_{j i}$ and thus $\max _{i j}\left|a_{i j}^{-1}\right|=c$.

[61] G. Hardy, J.E. Littlewood and G. Pólya, Inequalities (Cambridge University Press, Cambridge, 1952).

[62] W. Beckner, Ann. of Math. 102 (1975) 159.

[63] K.I. Babenko, Amer. Math. Soc. Transl. Ser. 244 (1962) 115. 
[64] E.H. Lieb, Invent. Math. 102 (1990) 179.

[65] M. Reed and B. Simon, Methods of modern mathematical physics. Vol. XI: Fourier analysis, selfadjointness, (Academic Press, New York, 1975).

[66] M.E. Andersson, The Hausdorff-Young inequality and Fourier Type, Ph. D. Thesis, Uppsala, 1993.

[67] A. Stam, Inform. Contr. 2 (1959) 101.

[68] A. Dembo and T.M. Cover, IEEE Trans. Inf. Theory, 37 (1991) 1501.

[69] O. Rioul, IEEE Trans. Inf. Theory, 57 (2011) 33.

[70] P. Grünwald, the Minimum Description Length principle (MIT Press, 2007).

[71] K. Kraus, Phys. Rev. D 35 (1987) 3070.

[72] E.H. Lieb, Adv. in Math. 20 (1976) 151.

[73] K. Yosida, Functional Analysis, (Springer Verlag, Berlin, 1968).

[74] M. Brune, E. Hagley, J. Dreyer, X. Maitre, A. Maali, C. Wunderlich, J. M. Raimond, and S. Haroche, Phys. Rev. Lett. 77 (1996) 4887.

[75] P.A. Knott, W.J. Munro and J.A. Dunningham, Phys. Rev. A 89 (2013) 053812.

[76] P.A. Knott, T.J. Proctor, K. Nemoto, J.A. Dunningham, W.J. Munro, arXiv: 1405.7198.

[77] C. Brukner and A. Zeilinger, Phys. Rev. Lett. 83 (1999) 3354.

[78] see, e.g., G. Wilk and Z. Wlodarczyk, Phys. Rev. A 79 (2008) 062108.

[79] G.H. Golub and C.F. Van Loan, Matrix Computations, 3rd ed., (John Hopkins University Press, Baltimore, 1996).

[80] G.W. Steward and J.-G. Sun, Matrix Perturbation Theory, (Academic Press, New York, 1990).

[81] I.I. Hirschman, Jr., Am. J. Math. 79 (1957) 152.

[82] B. Al-Saqabi, L. Boyadjiev and Yu. Luchko, Eur. Phys. J. Special Topics 222 (2013) 1779.

[83] N. Laskin, Phys. Lett. A 268 (2000) 298.

[84] D. Petz, Rep. Math. Phys. 23 (1986) 57.

[85] R. Renner, Security of quantum key distribution, Ph.D. Thesis, ETH Zurich, 2005.

[86] Note that these limits do not contradict the constraint $1 / t+1 / r=-2$. To see this we go back to the defining equation $1 /[2(1+t)]+1 /[2(1+r)]=1$ which is clearly satisfied for the simultaneous limits $t \rightarrow 0_{+}$and $r \rightarrow 0_{-}$. 